\title{
Structural Damage Detection Using Frequency Response Function
}

\author{
Arash sabzi ghasemkheili* \\ Department of Civil Engineering, Noshirvani University of Technology, Babol, Iran, \\ arash.sabzi@gmail.com \\ MohammadReza Davoodi \\ Department of Structural and Earthquake Engineering \\ davoodi@nit.ac.ir \\ mobin mohamadzade rad \\ Department of Civil Engineering, Noshirvani University of Technology, Babol, Iran, \\ Mobin_n95@yahoo.com
}

\begin{abstract}
The concession period of man-made structures is limited and not eternal under any circumstances. Various internal and external factors cause the components of the structure to be damaged and the structure to be in serious trouble and even completely destroyed under the service load. The identification of damage to a structure has become a matter of interest to many researchers in recent decades, because catastrophic destruction of structures can be prevented by early damage detection. In addition, the service life of the structures can be increased if the defects and damages are timely eliminated. In present study, the damage to a cantilever beam constructed in the laboratory is detected based on the data from frequency response function. To do this, changes in the stiffness and natural frequencies of a structure are considered as factors in searching for a damage to the beam. The present study is carried out in two empirical and analytical phases. In the empirical phase, a physical model of cantilever beam is developed according to the requirements for damage detection. Then, the modal testing is planned to obtain the empirical frequency response functions of the cantilever beam. In the analytical phase, damage is detected using a finite element model of cantilever beam and considering the damage as a vector (including position and intensity of damage) in the cantilever beam element stiffness matrix as well as solving an optimization problem using a genetics algorithm. After conducting modal analysis on a healthy and damaged beams and obtaining frequency response functions, a finite element model updating is performed to consider the behavior of the frame connected to the cantilever beam. It can be seen that the program written in the theoretical phase of damage detection is very accurate so that it can detects damage at several points and at different intensities. In the empirical phase, damage detection is presented in a sample damaged at one point and the program is able to accurately detect the position and intensity of the damage. In the scenario of being damaged at two different points, in addition to the detection of damaged elements, the method considers a small percentage of damage for the healthy elements. In general, the proposed method is an accurate and appropriate method for detecting the damage to the cantilever beams.
\end{abstract}

Keywords: damage detection, frequency response function, optimization, modal analysis, genetics algorithm, updating

\section{Introduction}

A structure may be damaged, due to a change in the use, or sudden loading such as wind and earthquakes, over time. Additionally, the structure materials erode and deteriorate due to the environmental conditions over time, resulting in the change in their properties. Significant damage to a structure is often be found as changes in physical properties, such as decreased stiffness of the structure and corresponding changed natural frequencies. If damage is not timely monitored and eliminated, it can affect the performance of the structure, increase maintenance costs, and cause the collapse of the structure in an unfortunate event [1]. Therefore, from performance and safety viewpoints, it is necessary and useful to know some methods for early damage detection. To this end, structural damage identification is now a necessary component of the new engineering disciplines and known as the Structural Health Monitoring (SHM). Health monitoring involves the observation of a structure over time using dynamic response measurements to determine the "health" or "proportionality" of structures under gradual or sudden changes in their condition.

Nowadays, structural health monitoring, as interdisciplinary science, has become significant in identifying damage and determining the service life of structures, because early damage detection can prevent catastrophic collapse of the structure. In the case of earthquake zones, where local damage of structural elements can lead to overall damage, determining the position and intensity of the damage becomes very important. Perceptible 
benefits of health monitoring for users include better performance prediction, lower service cost and a more reliable structural safety assessment [2]. As it is increasingly accepted that there is a need to monitor the response, more efficient and powerful algorithms are needed to extract useful information from a great deal of collected data. Structural damage detection methods are divided into two groups: 1. Static methods and, 2. Dynamic methods [2]. To identify and detect structural damage, using the dynamic response is usually preferred to the static response, because the dynamic signals provide more information and can prevent non-uniqueness of the response. Unlike static measurements, in dynamic measurements, the effect of noise can usually be eliminated using low-pass filters. In addition, performing dynamic measurement through accelerometers is easier than static measurement [2].

The theoretical basis of dynamic methods lies in the fact that damage and failure cause changes in the dynamic properties of the structure. These dynamic parameters characterize the status of the structure. Many people have been interested in detecting damage without the need to deconstruct the structure using frequency responses in the last few decades, because any changes in the physical properties of structures such as stiffness, mass and damping due to damage, change the frequency response of the structure. The underlying principle of most damage detection methods is that the damage to the structures changes the properties, such as stiffness, the mass, and the energy dissipation, which are obtained using the measured dynamic response of the system. Such an identification can be done to take precautionary measures to plan for maintenance of the structure, if necessary. Traditionally, visual inspections are carried out to identify damage to building structures, but today, due to the big size and importance of structures, visual inspections are costly, time consuming and impractical. Therefore, it is necessary to utilize indirect methods which can continuously inspect the structure to detect its problems before it reaches a critical state [2]. In present study, damage to a cantilever beam is detected using the data from the frequency response function and the genetic algorithm. To do this, in the first step, a model updating of a cantilever beam is performed using a laboratory sample, and in the next step, the error of the analytical responses derived from the finite element models of healthy and damaged structures is minimized in order to determine the position and intensity of the damaged elements.

\section{Health monitoring}

The structural health assessment process is called "Structural Health Monitoring" (SMH) [1]. In monitoring the structure, it is attempted to measure the inputs and responses of the structure in order to use them in an inverse analysis to derive the properties of structure and track their changes and thereby detecting the onset of damage under the structural conditions. Then, numerical modeling of the structure and its re-analysis regarding the existing conditions are performed to evaluate the structural efficiency, in order to retrofit it if necessary. Therefore, the SMH process of a structural system is to monitor the system by measuring the dynamic responses through sensors, to extract the properties sensitive to structural damage using the measurement results, and to analyze those properties to determine the current system health status [1]. In a long-term SHM, the output of this process periodically updates the information on the ability of the structure to execute its intended performance. Structural health monitoring refers to a process used for obtaining accurate momentary information about the condition and performance of the structure. The main purpose of monitoring is to reveal the unusual behavior of the structure, which indicates the undesirable condition of the structure. The data obtained from the monitoring are used to optimize the performance, maintenance, repair and replacement of the structure based on the reliable and measured target data. In the discussion on the health monitoring, damage is defined as changes occurring during the exploitation of the structure. Deubling et al. [3] divide the damage detection methods into three levels:

1. Those methods that identify the damage.

2. Those methods that, in addition to the damage, determine its position.

3. Those methods that, in addition to the damage and its position, determine its intensity.

The method followed in present study is of the third group and, in addition to the damage and its position, it can determine its intensity.

The SHM process has several stages. In one of them, the inherent properties of the structure are extracted. In fact, this stage is carried out to identify the damage-sensitive properties which are obtained using the measured vibration response of the structure, allowing the detection of healthy and damaged structures. The properties are selected based on the past experience as well as the measured information on the system. After extracting frequency responses by tracking changes in these responses, damage to the structure can be detected using a series of methods. Once the damage is identified, the structure should be analyzed considering the existing status and performance of the structure should be measured and, some measures must be taken into account for repairing and retrofitting the structure, if necessary [2]. 


\section{Method}

Detecting the damage to a cantilever beam using the finite element model updating and using the dynamic properties of the cantilever beam as a response in the damage detection process is one of the important parts of the research. These dynamic properties, which their empirical and analytical values are compared and the differences between them are minimized, are treated as natural frequencies. The empirical phase of damage detection process includes following steps: (1) development of a physical model; (2) empirical measurement; (3) extraction of empirical responses. This phase is planned in a way that the natural frequencies of a cantilever beam is obtained in the third step. As mentioned, in present study, damage detection is carried out using one of the outputs of the frequency response function and updating the stiffness matrix in the Matlab Software. In present study, first, two empirical and analytical phases and how to extract the empirical and analytical responses are fully described. In the next section, the finite element model of the beam, the mass and stiffness matrices are explained, and it is shown that how damage is considered in the stiffness matrix. Then, an optimization problem and how to solve it to reduce the difference between empirical and analytic responses are explained. Finally, it ends with a complete explanation of the algorithm used in present research (the genetic algorithm).

\subsection{Empirical Phase}

\subsubsection{Test and Measurement}

Modal testing is used to derive the empirical responses of the structure in the updating process. Modal testing is an empirical technique used for obtaining a modal model of a linear time-invariant vibrating system. The theoretical basis of this technique lies in the relationship between the vibration response at a point of the structure and being stimulated at the same or another point as a function of the stimulation frequency. This relationship is often referred to as a complex mathematical function, called the Frequency Response Function (FRF). A complete set of FRFs are obtained considering different combinations of stimulation points and responses on the structure. Modal testing involves measuring these FRFs.

\subsection{Analytical phase}

\subsubsection{A Finite element model for a bending element}

Dynamic properties of the structure are required to detect damage. To determine these properties, a finite element model of the structure is required. in the following, it is explained how to determine the finite element model of a bending beam. To analyze the finite elements, the first step is to divide the object into smaller elements. In this method, the continuous environment is divided into simple and smaller geometric elements called "finite element". This doing is called " discretizing" and the common point between the elements is called "joint". The smaller the length of the element, the higher the accuracy of the analysis. In most linear structures such as beams, the joints are placed at the beginning and end of an element. The degree of freedom of a joint is the number of possible displacements in it. The degrees of freedom of joints are obtained by determining the position of a joint and examining the freedom of its independent movements, the degrees of release of those nodes are determined. The degree of freedom of an element is determined by summing up the degrees of freedom of the joints of that element.

Fig. 1 shows a bending element. The finite element model of a beam is composed of two-joint elements. There are 3 degrees of freedom in each joint. Since the axial force effect is negligible, the effect of axial force and consequently, changes in mode shapes are neglected. Each one-dimensional bending element in each joint has two degrees of freedom. Here, joint displacements of $q_{1}$ and $q_{3}$ denote the transfer along the vertical axis y and joint displacements of $q_{2}$ and $q_{4}$ denote the rotation around the z-axis, which is perpendicular to the plane.

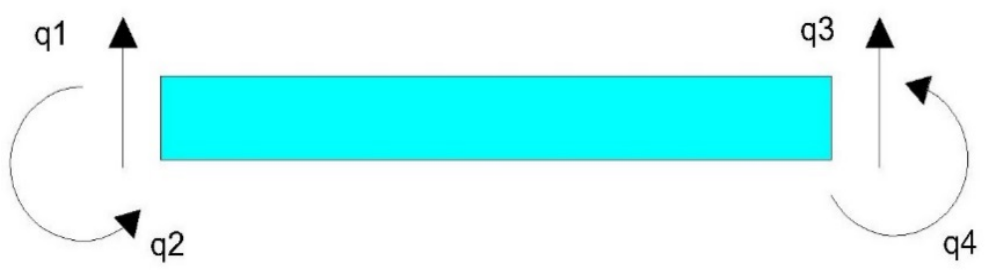

Figure 1. Bending element

Since the number of possible displacements represents the number of unknowns, the number of degrees of freedom of a system is, in fact, the number of simultaneous equations that must be solved. In addition, in structural analysis problems, a series of joints are bound to the required axes to prevent rigid motion in the structure. By this description, the number of simultaneous equations that must be solved in order to determine the unknowns of a structure is equal to the total number of degrees of freedom of the system minus the number of bound axes. 


\subsection{Results of the modal testing}

\section{Results of analysis}

A modal testing is applied to a steel cantilever beam in the clamped condition. In modal testing models, this type of connection is usually considered using retaining clamps. However, due to the dimensions of the tested beam, using such claps does not provide the right clamped conditions. In order to create the clamped condition, the test box beam is welded to a rigid frame from all four directions. As shown in Fig. 2, the box beam is connected to the desired frame by welding.

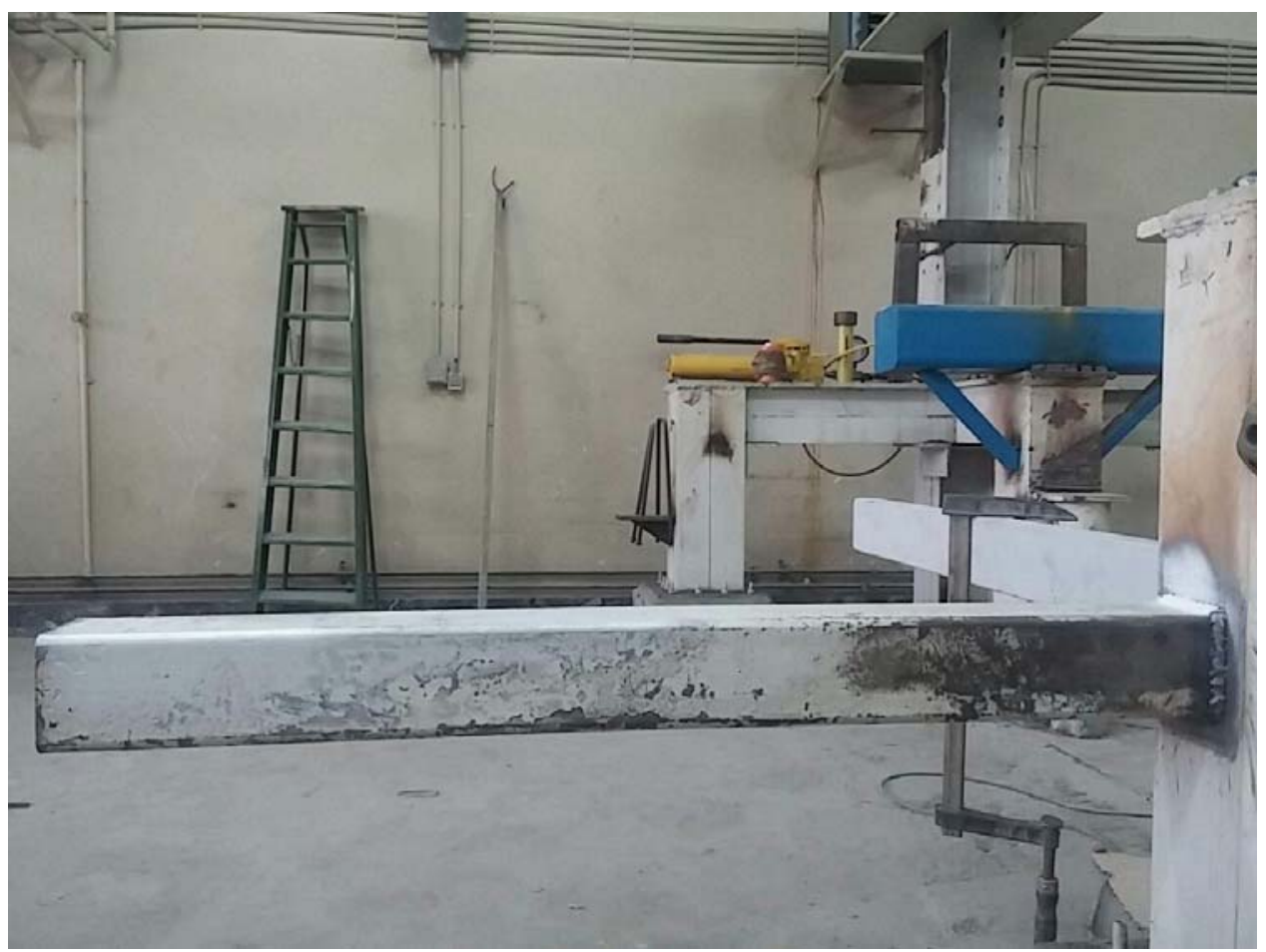

Figure 2. The test cantilever beam

In this test, five points of the beam, also defined in the Pulse LabShop software, are stimulated by the hammer, as shown in Fig. 3. It should be noted that defining the points in the Pulse LabShop software is only intended to expedite the testing process. In Fig.3, the black spot is the position stimulated by the hammer and the red spot shows the position of the accelerometer.

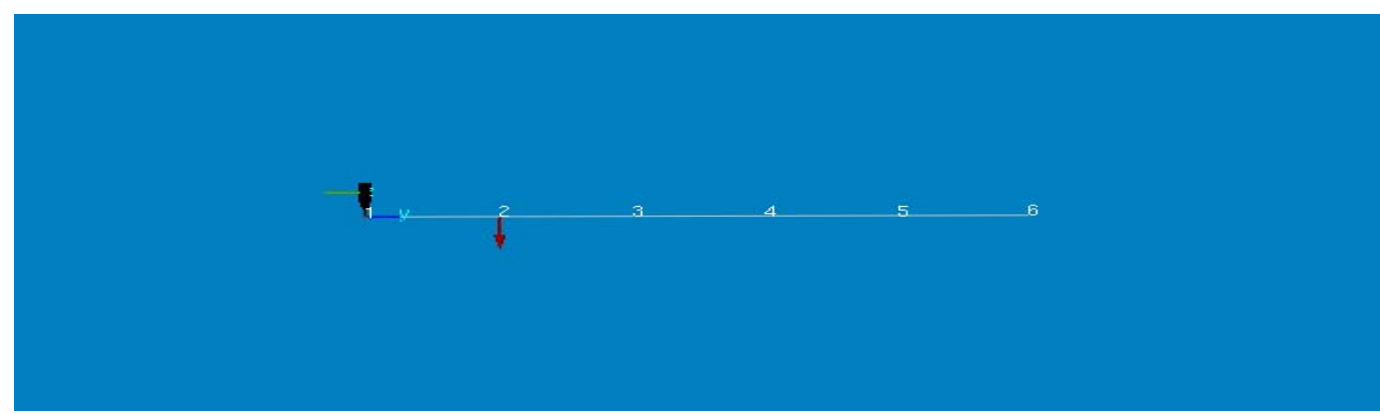

Figure 3. A view of the model developed in the Pulse Labshop software

\subsection{Stimulation mechanism}

In Fig. 4, three examples of the frequency response function are shown with FRF3 brown, FRF3 red and FRF3 black. It is observed that the responses obtained with different pieces have different qualities. 


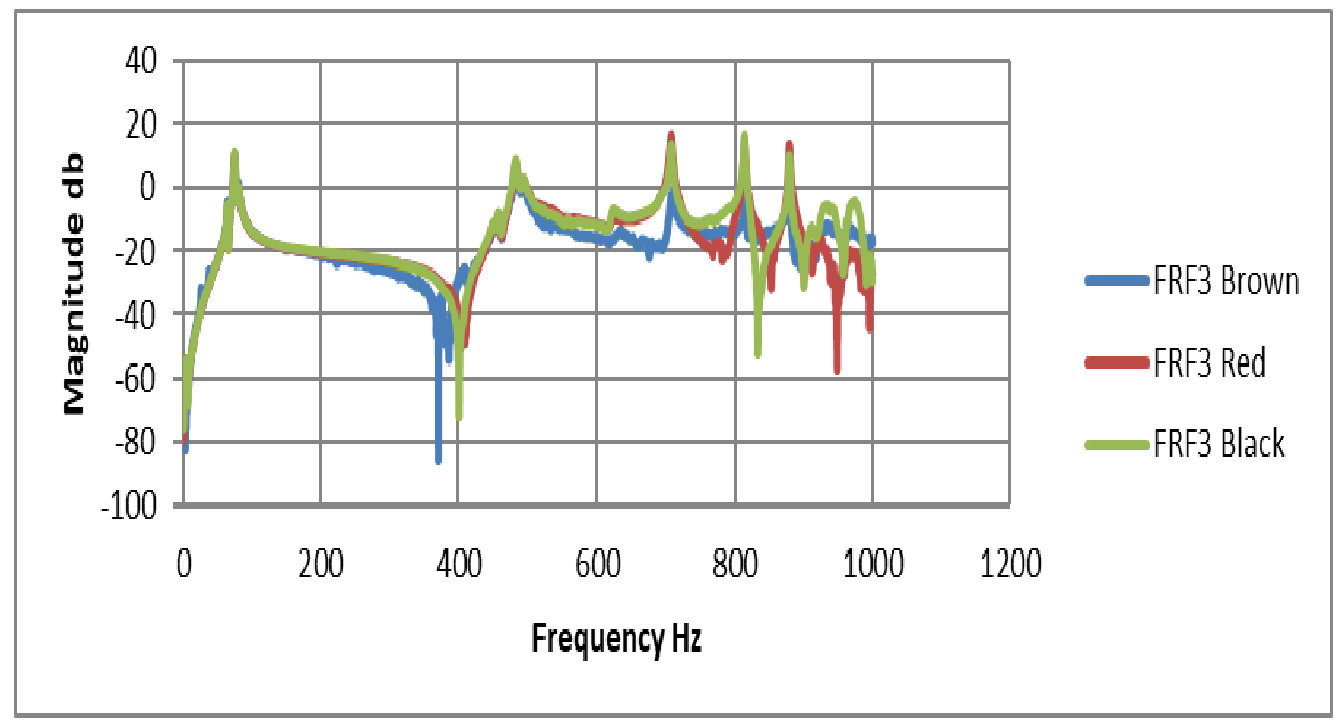

Figure 4. Comparison of the qualities of the frequency responses at the third point of the healthy beam

\subsection{Examination of Linearity}

Fig. 5 represent the frequency response function of the second point of the beam for two different amplitude of force applied to the cantilever beam.

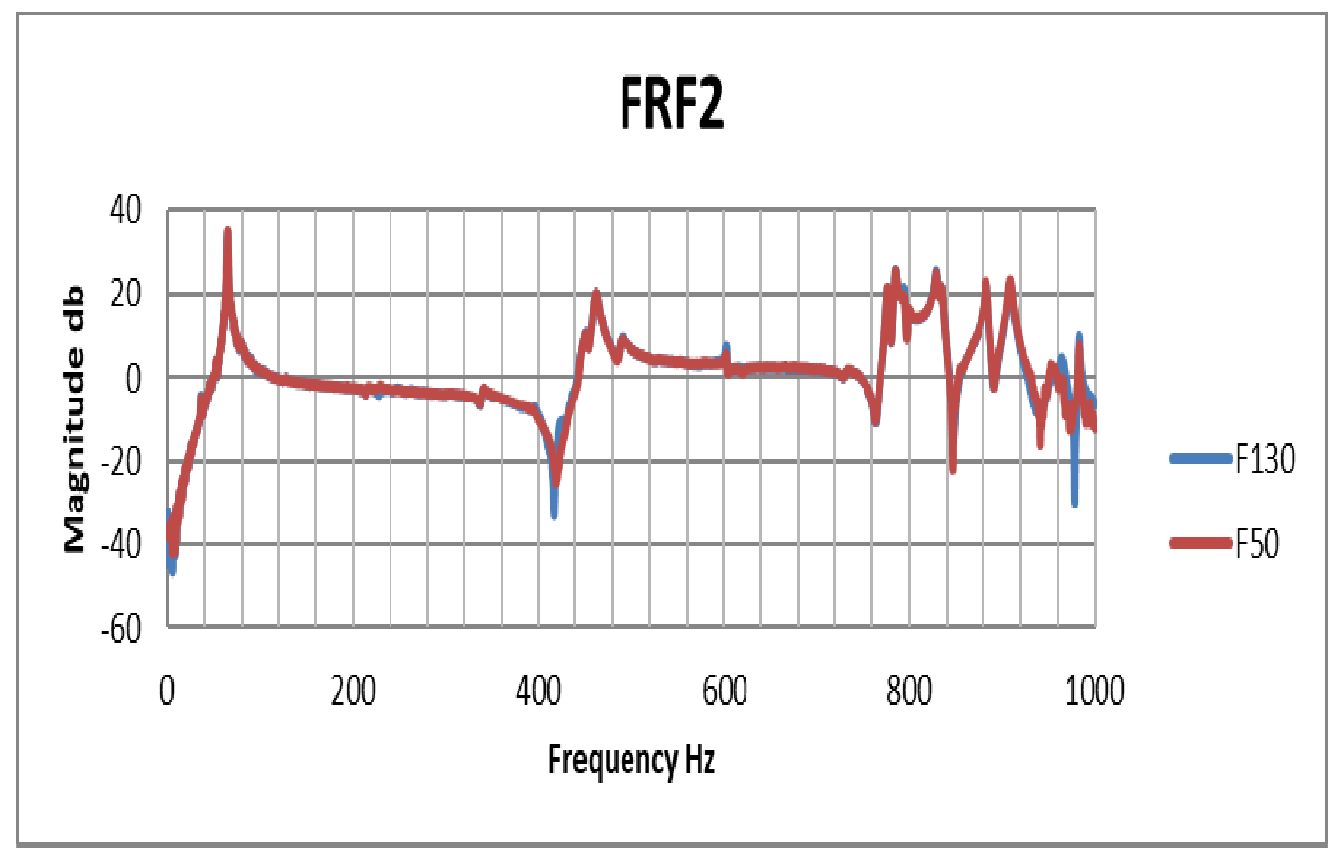

Figure 5: Examination of the linearity

The results of two 130 and 50N hammer blows in the frequency range of 0-400 Hz are very close to each other. In the frequency range of 400-1000, there is a very small difference between them, and the only significant difference is in the amount and size of the anti-resonance frequency. Since the position of anti-resonance frequency is local, it is concluded that the beam has a negligible non-linear behavior in the range of exerted force.

\subsection{Examination of Repeatability}

Repeatability of the frequency response function for the cantilever beam is shown in Fig.6. 


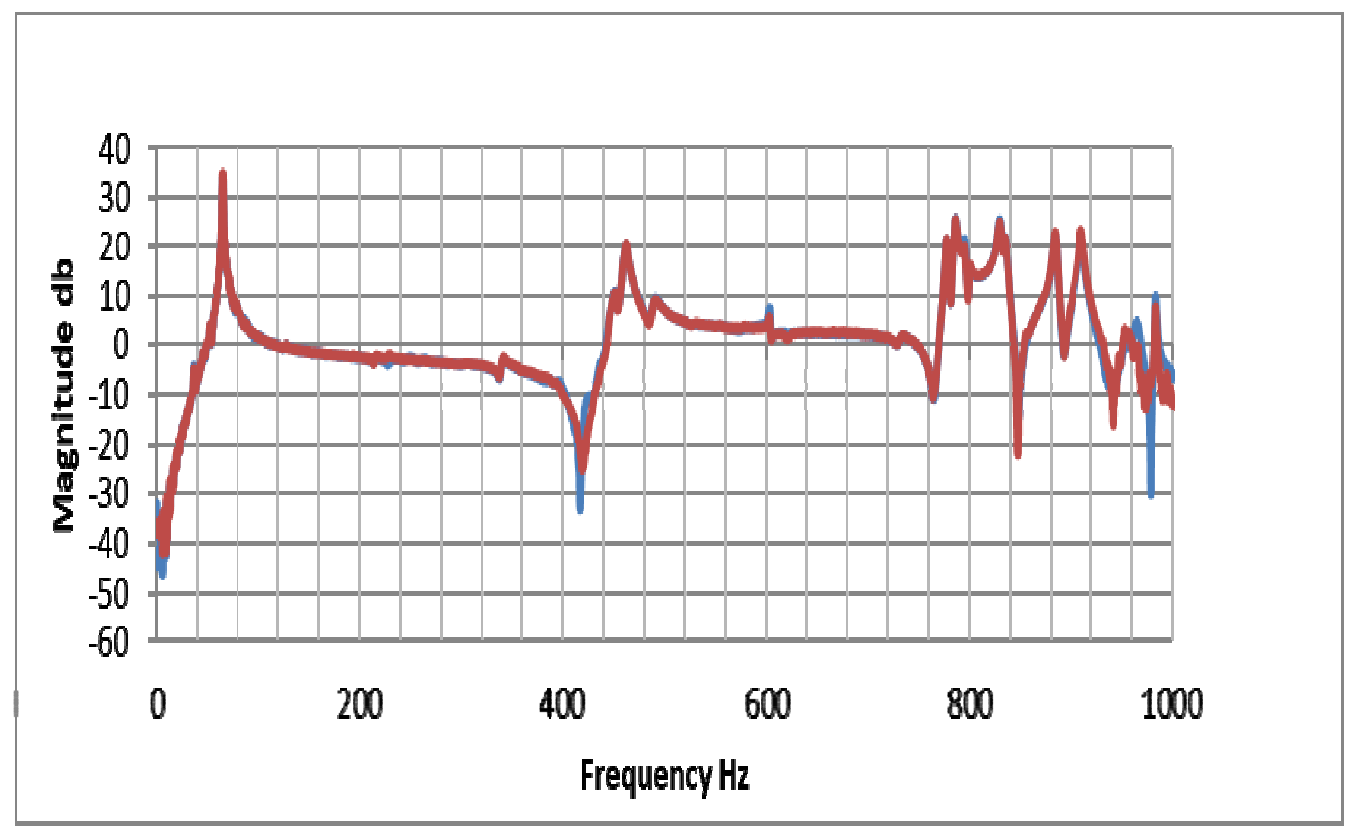

Figure 6. Examination of repeatability

The above diagram shows two frequency response functions measured on 27 September 2018 (red curve, at about $22{ }^{\circ} \mathrm{C}$ ) and 11 November 2018 (blue curve, at about $13^{\circ} \mathrm{C}$ ). Comparing these two frequency response functions shows that the repeatability of the measurements is very favorable in the same environmental conditions, and no significant changes in the behavior of the structure or the test conditions have been made during the test.

\subsection{Examination of reaction}

Fig.7 shows the frequency response function of the second point which is developed by the hammer blow at the first point and the frequency response function of the first point which is developed by the hammer blow at the second point. In the graph, the first and second numbers represent the positions of the blow and the accelerometer, respectively.

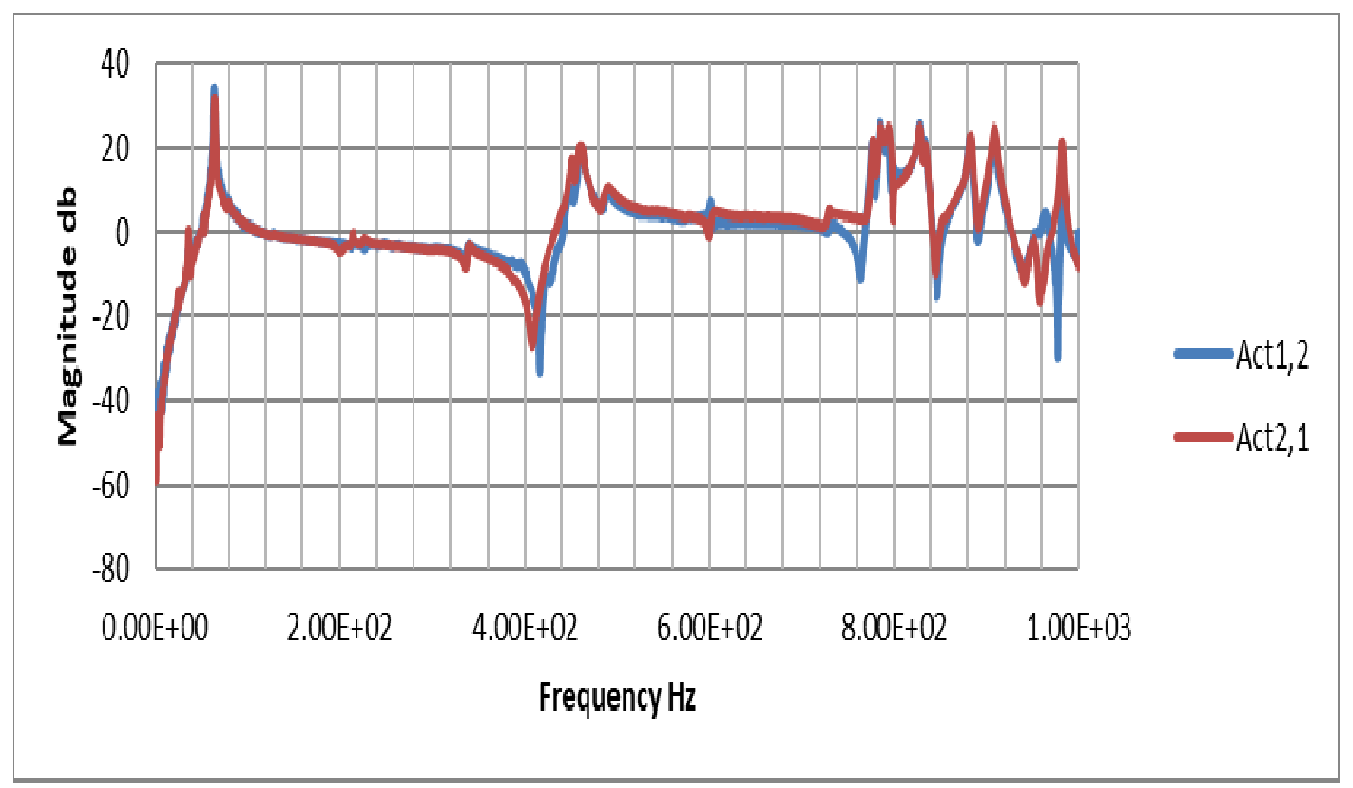

Figure 7. Examination of reaction

As shown in above graph, the frequency response functions are very similar to each other at two symmetric points of the FRF matrix and the only difference between them is observed in the anti-resonance positions. 


\section{6-1-2-4. Output of Pulse labshop Software}

The Pulse software gives a frequency response function graph, as a main output, and dependency function and fuzzy changes graphs by stimulating each point. The dependency graph refers to the quality of the measured data, such that at peak points, the values are close to 1 and at non-peak points, they are far away 1 . An example of the frequency response function graph is shown in Fig.8 and Fig.9 shows the dependency function graph.

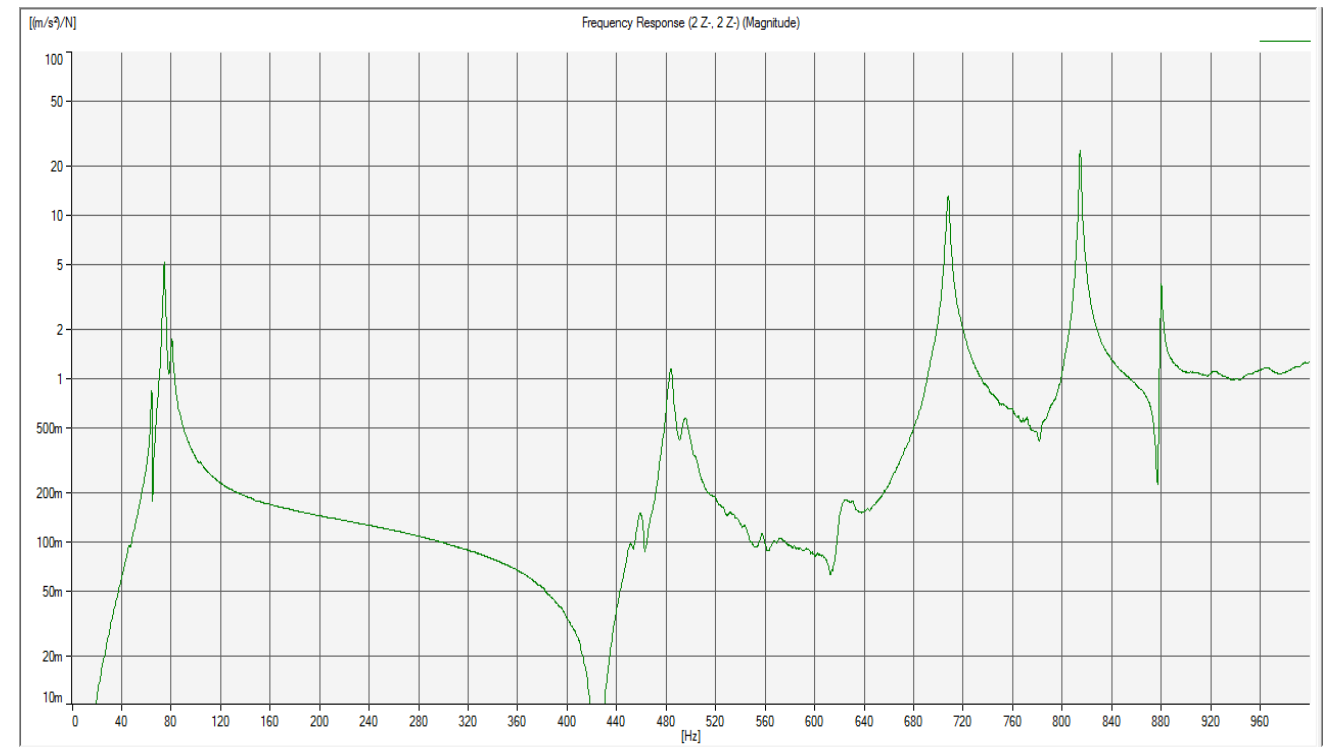

Figure 8. An example of the frequency response function obtained for a healthy beam

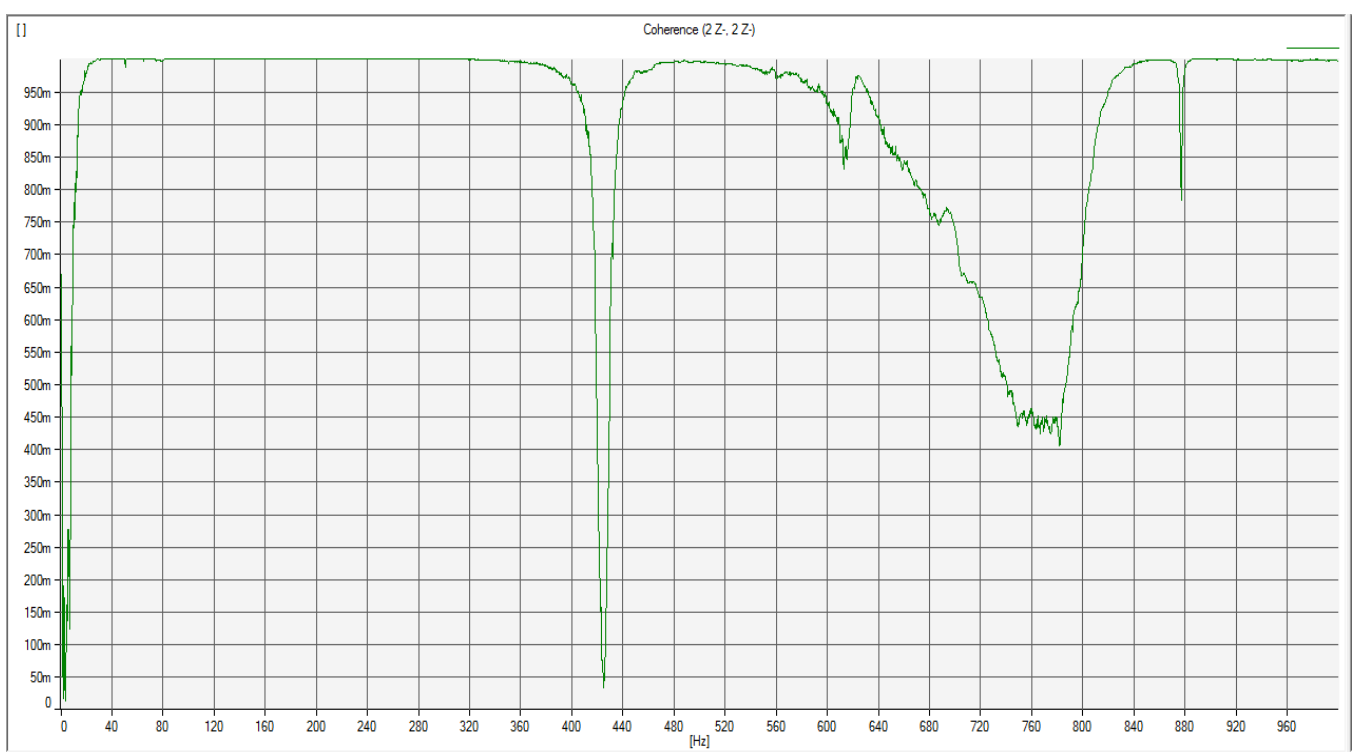

Figure 9 - An example of the dependency function

\section{Modal Analysis of a Healthy Beam}

In this section, using each frequency response function, the natural frequencies are extracted in three ways. First, an output is obtained for each point from the data of the frequency response function graphs in the Pulse software, and FRF graphs are plotted in Excel software. Then, in the ICAT software package, the normal frequencies of a healthy cantilever beam are obtained. 


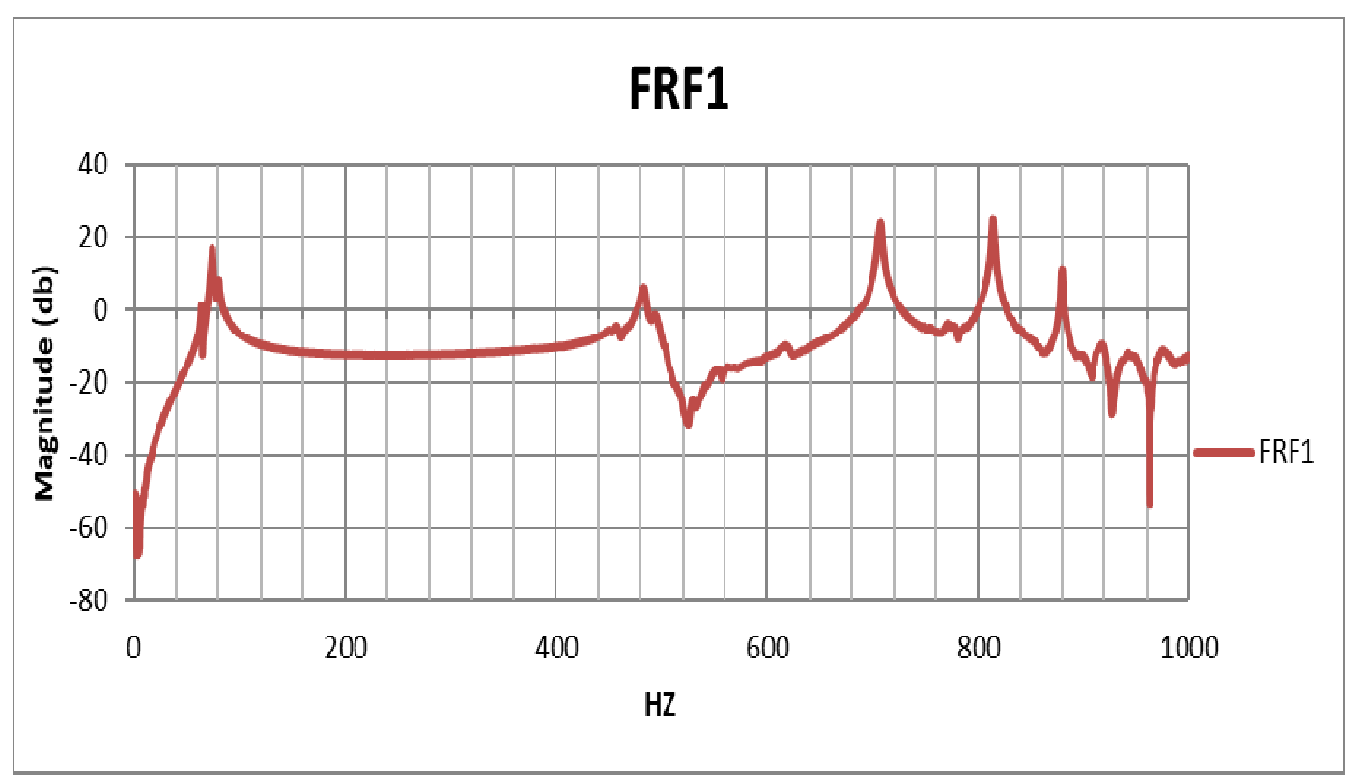

Figure 10 - Frequency Response Function of the first point on the healthy cantilever beam

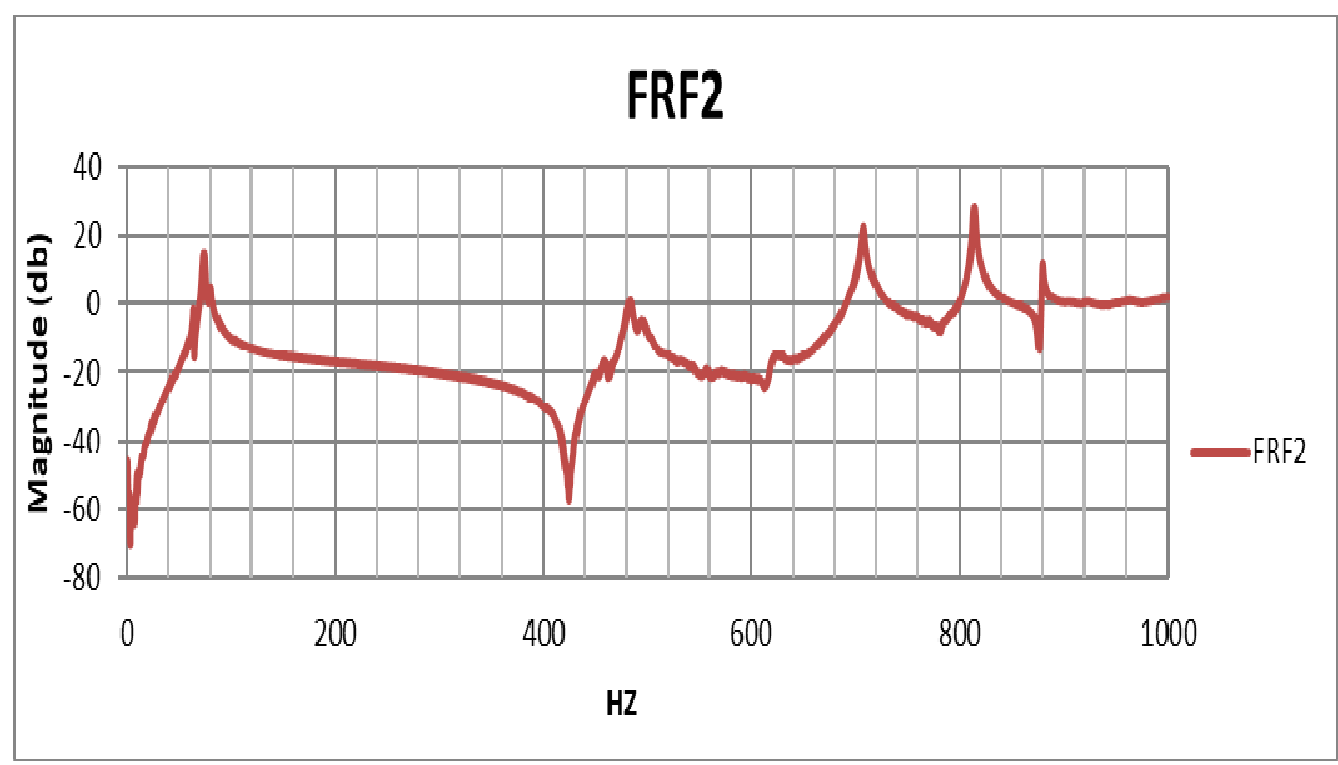

Fig. 11: Frequency Response Function of the second point on the healthy cantilever beam

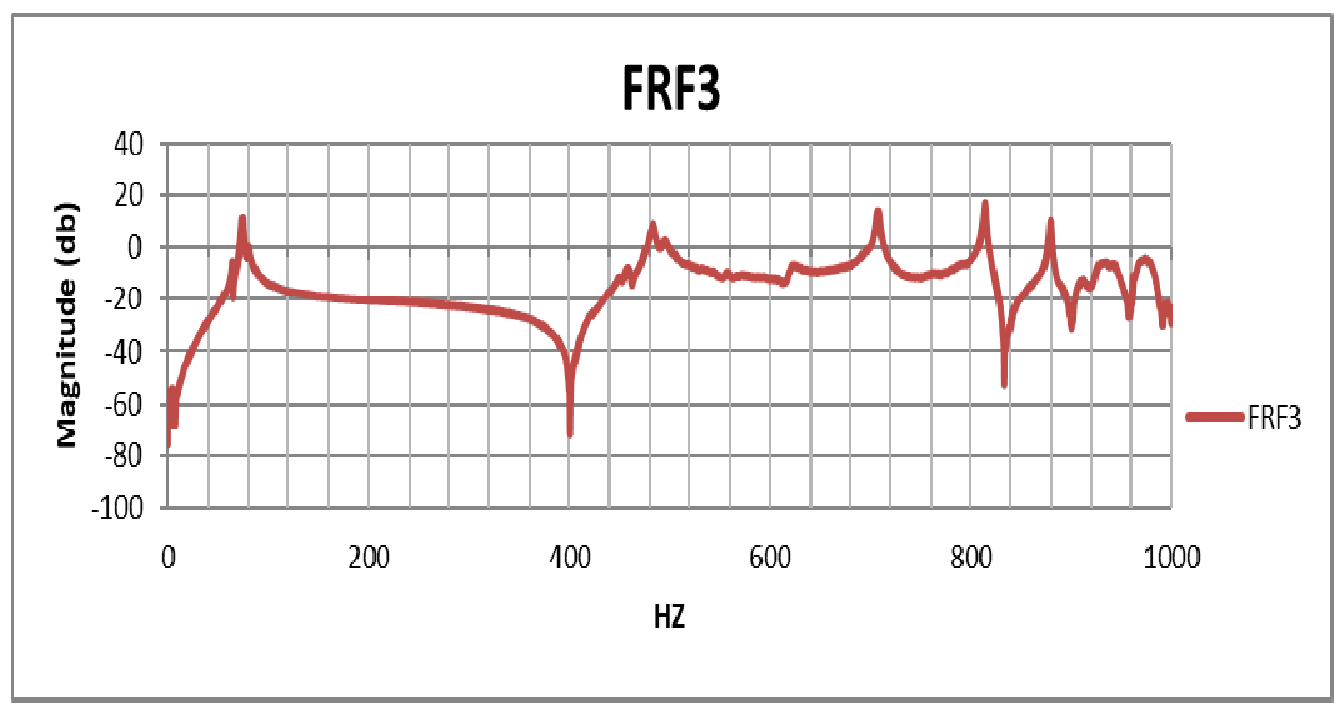

Fig. 12: Frequency Response Function of the third point on the healthy cantilever beam 


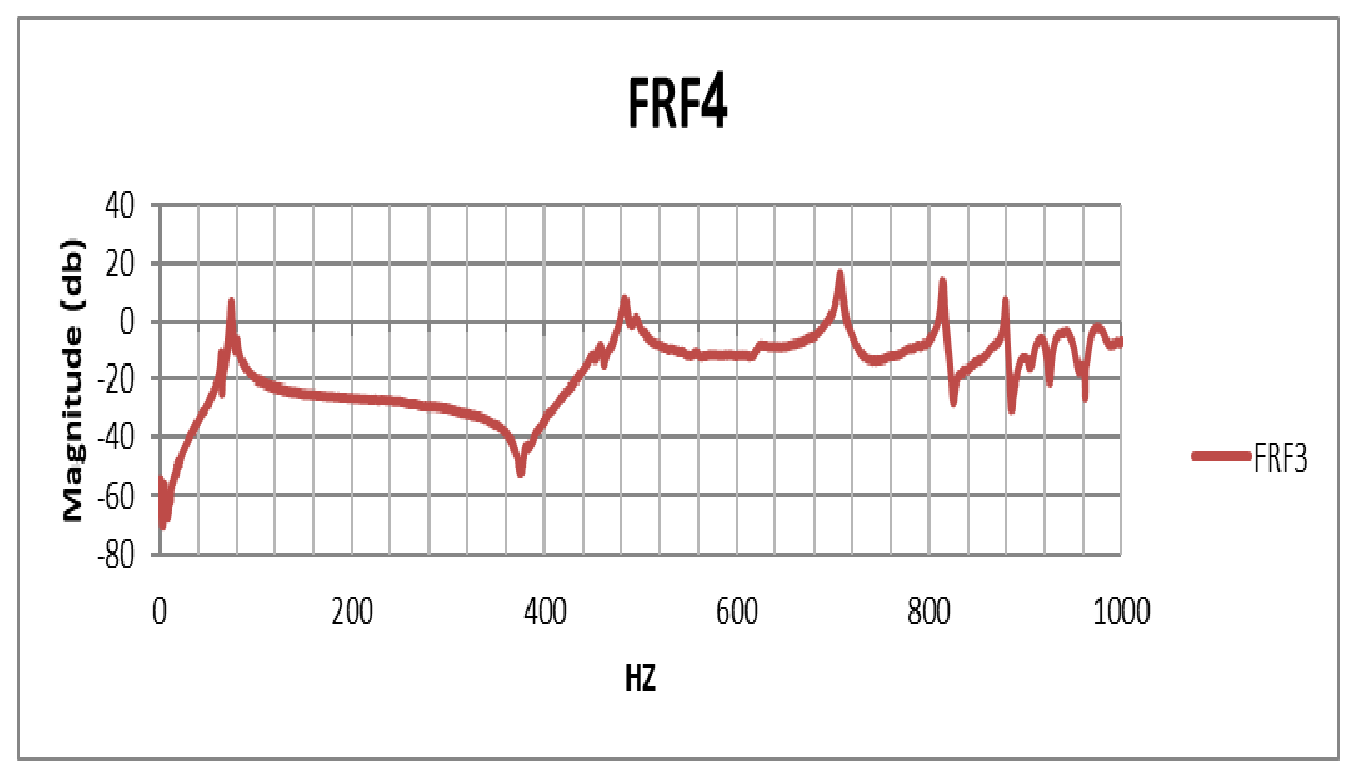

Figure 13 - Frequency Response Function of the fourth point on the healthy cantilever beam

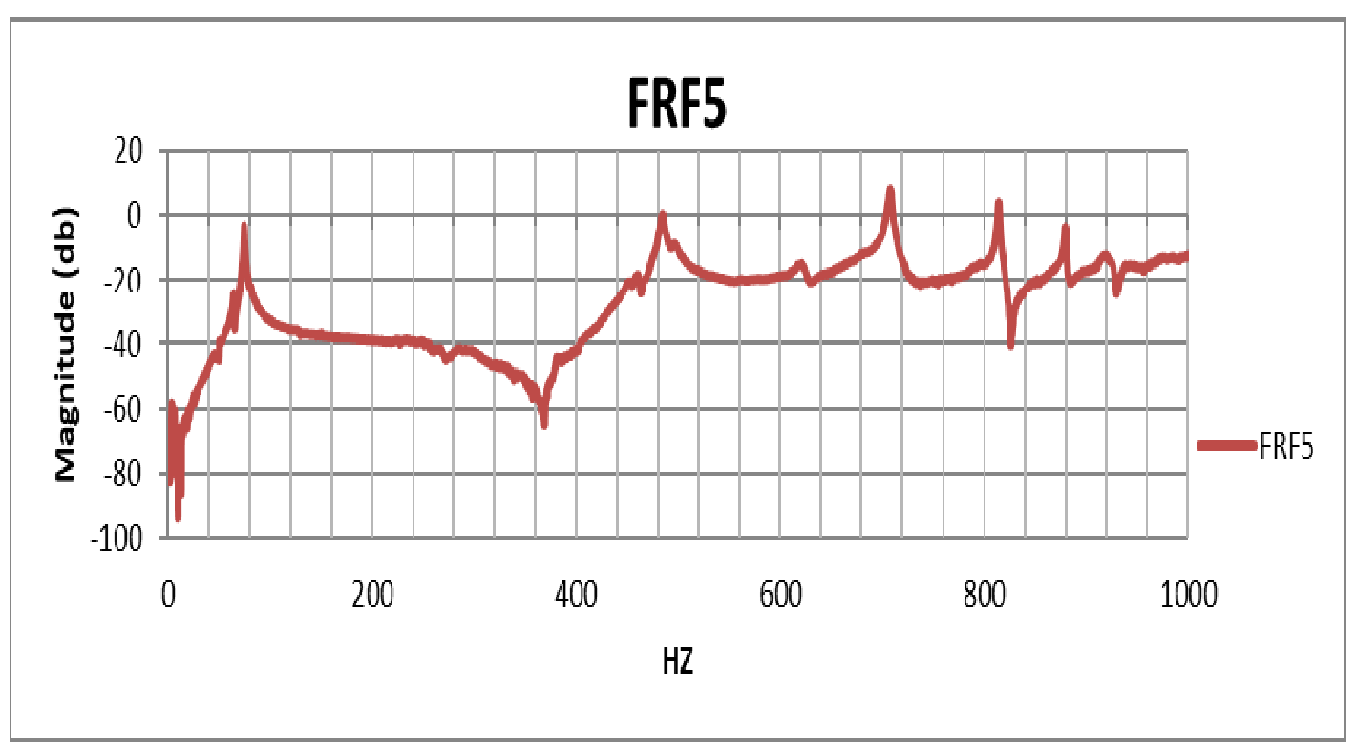

Fig. 14 - Frequency Response Function of the fifth point on the healthy cantilever beam

After obtaining the output of Pulse software, the data are entered into the MODENT software and the modal analysis is carried out. For example, Figure 4-5 shows the third, fourth, and fifth nodes of the cantilever beam simultaneously extracted using the NLLS1 technique (multi-functional multi degrees of freedom). 


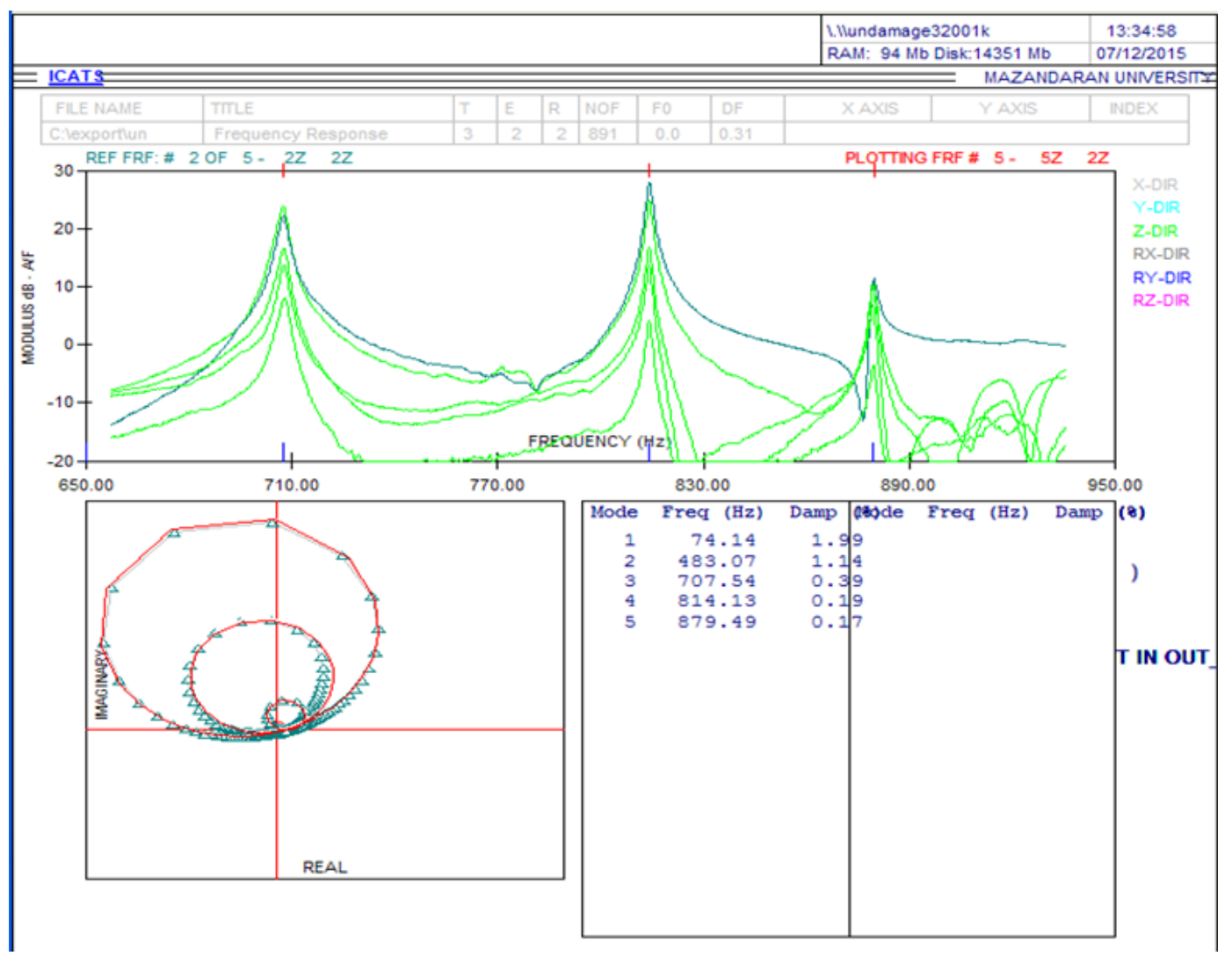

Figure 15: The simultaneous detection of the third, fourth, and fifth modes using the NLLS1 technique

In the upper rectangular box, the five frequency response functions measured considering the frequency response function of the second point as a reference are shown in a mode graph and in a frequency range of 650950. In the bottom left box, the Nyquist plot of one of the empirical functions (blue dotted line), along with its calculated theoretical function (red solid line) are shown in the same frequency range. Because of the existence of three modes, the plot consists of three circles. This indicates the desirable agreement of empirical and theoretical functions and the estimation of the modal parameters is correct. In order to implement abovementioned methods, the sub-program of MODENT, i.e. the ICATS package is used. although, it is possible to analyze the entire frequency domain in one step, according to the program tutorial, in order to achieve the maximum accuracy, the frequency domain is selected in such a way that there are three modes in it. Another example is shown in Fig.4-6. It shows the extraction of third mode using the GRFM method. 


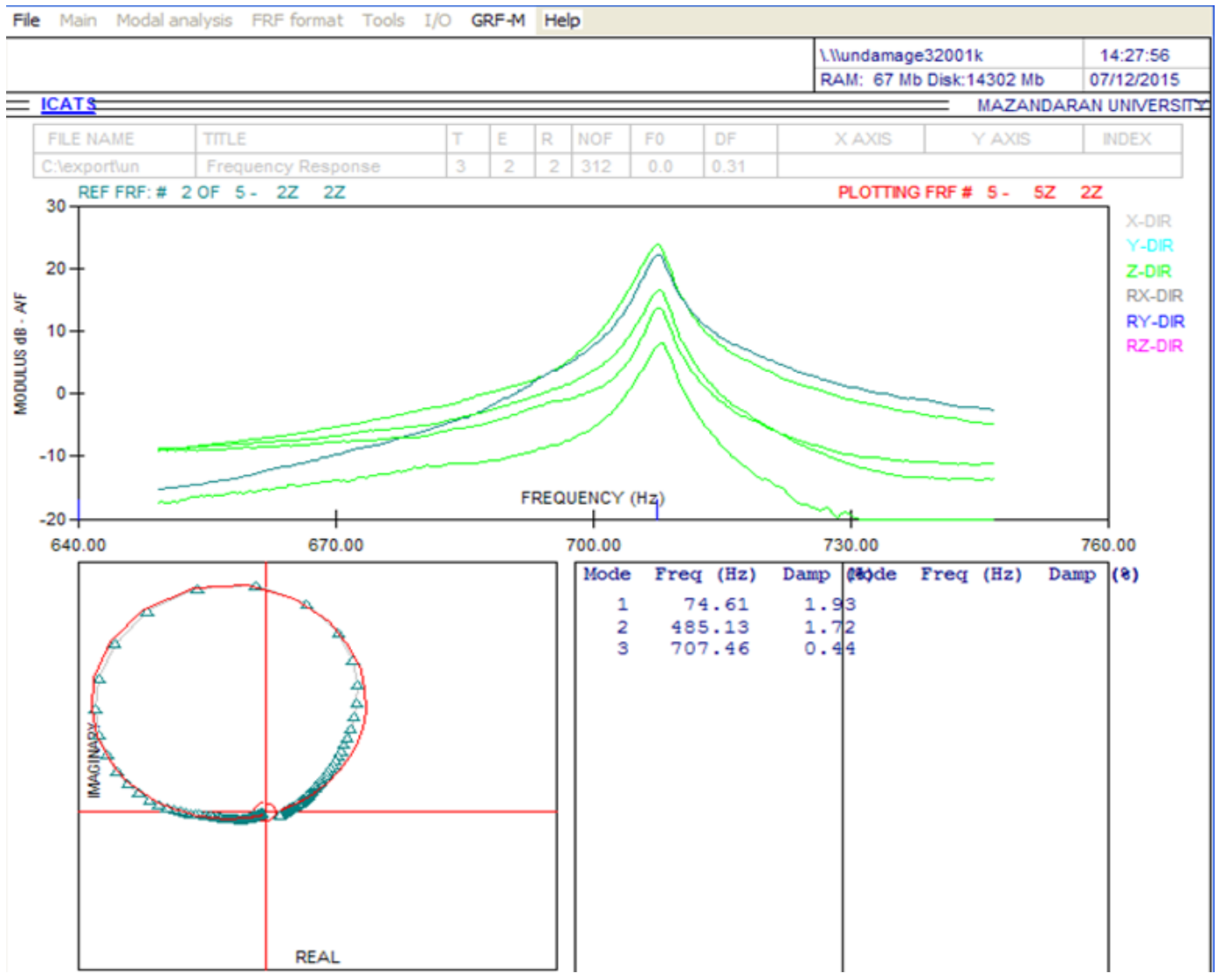

Figure 16 - Extraction of the third mode using the GRFM method

In the selected frequency range of 670-750, there is one mode and its corresponding Nyquist plot is a circle. The results of the health cantilever beam modal analysis performed using the NLLS1 technique are summarized in Table 12. The natural frequencies obtained using the NLLS1, NLLS2, and GRFM methods are highly consistent. The consistency of these frequencies ensures that the empirical phase of present study is done correctly.

Table 1: Results of empirical modal analysis of a healthy beam using three NLLS1, NLLS2, and GRFM methods

\begin{tabular}{|l|l|l|l|l|}
\hline Mode No. & NLLS1 & NLSS2 & GRFM & Considered \\
\hline & Frequency & Frequency & Frequency & Frequency \\
\hline 1 & 74.14 & 73.85 & 74.61 & 74.2 \\
\hline 2 & 483.07 & 483.09 & 485.13 & 485.76 \\
\hline 3 & 707.54 & 707.65 & 707.46 & 707.54 \\
\hline 4 & 814.49 & 813.12 & 814.16 & 813.92 \\
\hline 5 & 879.49 & 879.38 & 879.47 & 879.44 \\
\hline
\end{tabular}

\section{Results of modal updating}

Providing clamped conditions for a cantilever beam is always associated with uncertainties, resulting in the inconsistency of the finite element model and the empirical model. The difference between the natural frequencies obtained from the modal testing and those frequencies obtained from the model shows this incontinency. According to the previous section, in present study, finite element model updating is carried out to minimize this difference. The finite element model updating process in a dynamic mode, is repeatedly performed under the same condition by minimizing the difference between its five natural analytical and empirical frequencies and the highest obtained fitness is considered. 
Table 2. Natural frequencies obtained from the updated finite element model

\begin{tabular}{|l|l|l|l|}
\hline Mode No. & Empirical model & Updated model & Error (\%) \\
\hline 1 & 74.14 & 73.37 & 1.03 \\
\hline 2 & 485.12 & 489.32 & 0.86 \\
\hline 3 & 707.54 & 725.32 & 2.45 \\
\hline 4 & 814.12 & 832.68 & 2.16 \\
\hline 5 & 879.48 & 898.32 & 2.10 \\
\hline
\end{tabular}

\subsubsection{Modal Analysis of a Damaged beam}

To identify the damage in this cantilever beam, two damage scenarios are defined and different percentages of damages to the beam are considered. The results of the damaged beam modal analysis with the first and second scenarios are shown in the graphs and tables below.

\section{1-4-2-4 Scenario I: Damage to the second element}

To do this, first, the position of the damage is determined and then, as shown in Figs. 4-7, the damage is perpendicularly applied to $2 \mathrm{~cm}$ of a shear box beam height. The following graphs show the results of the modal test performed on the damaged beam.

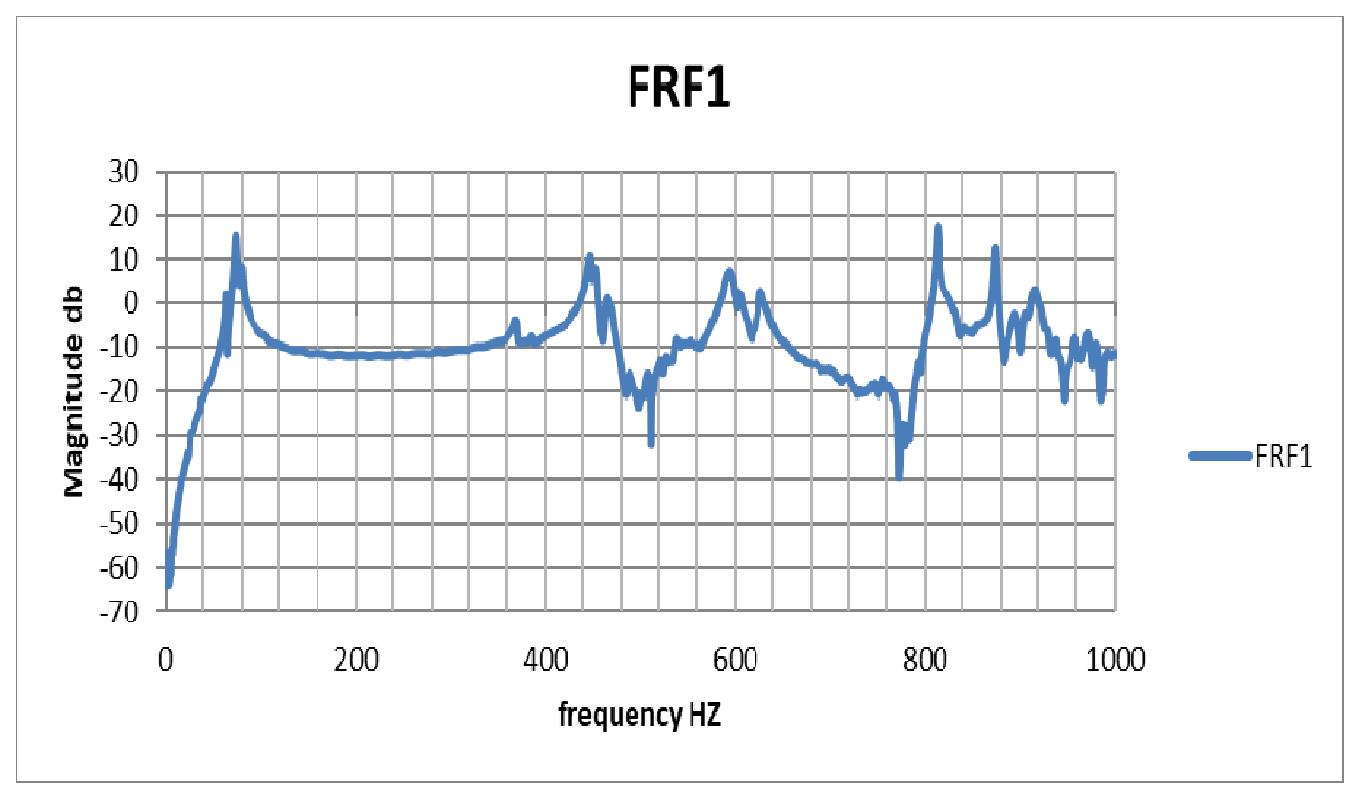

Figure 17 - Frequency response function of the damaged beam at the first point

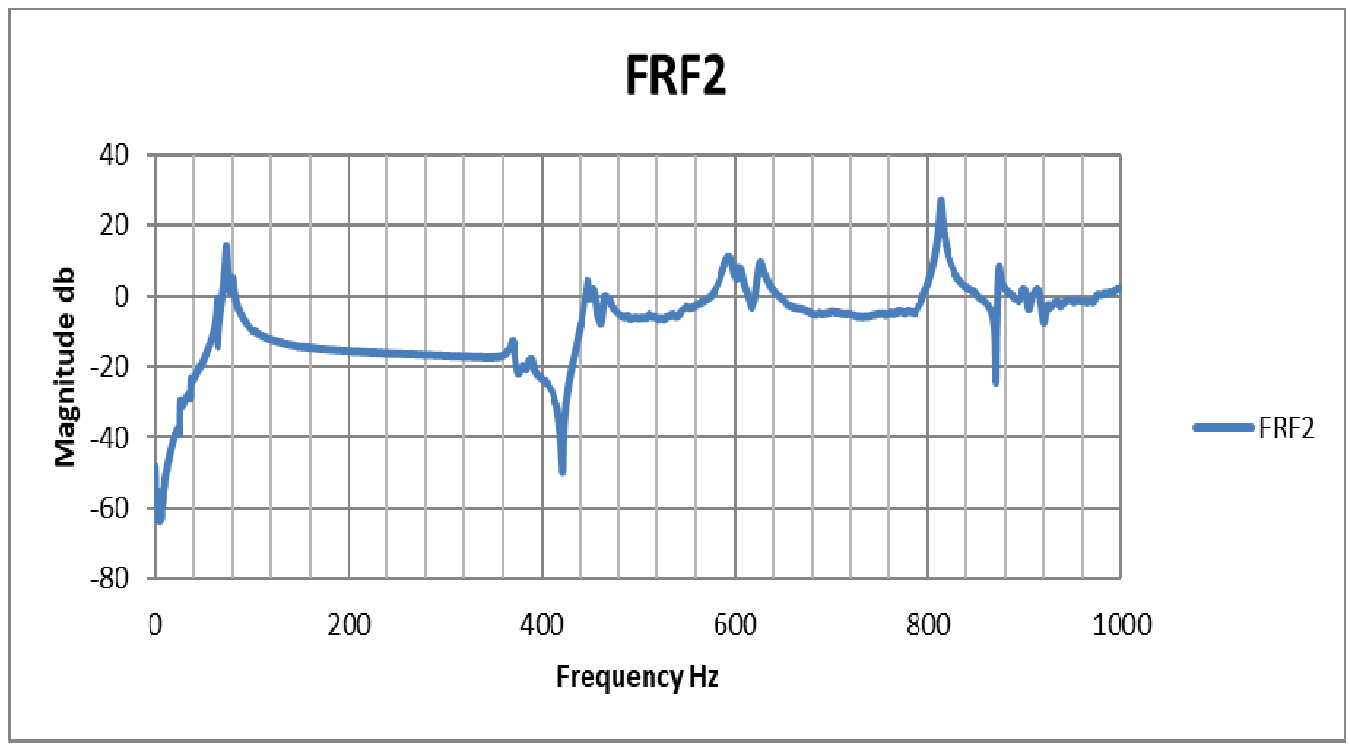

Figure 18 - Frequency response function of the damaged beam at the second point 


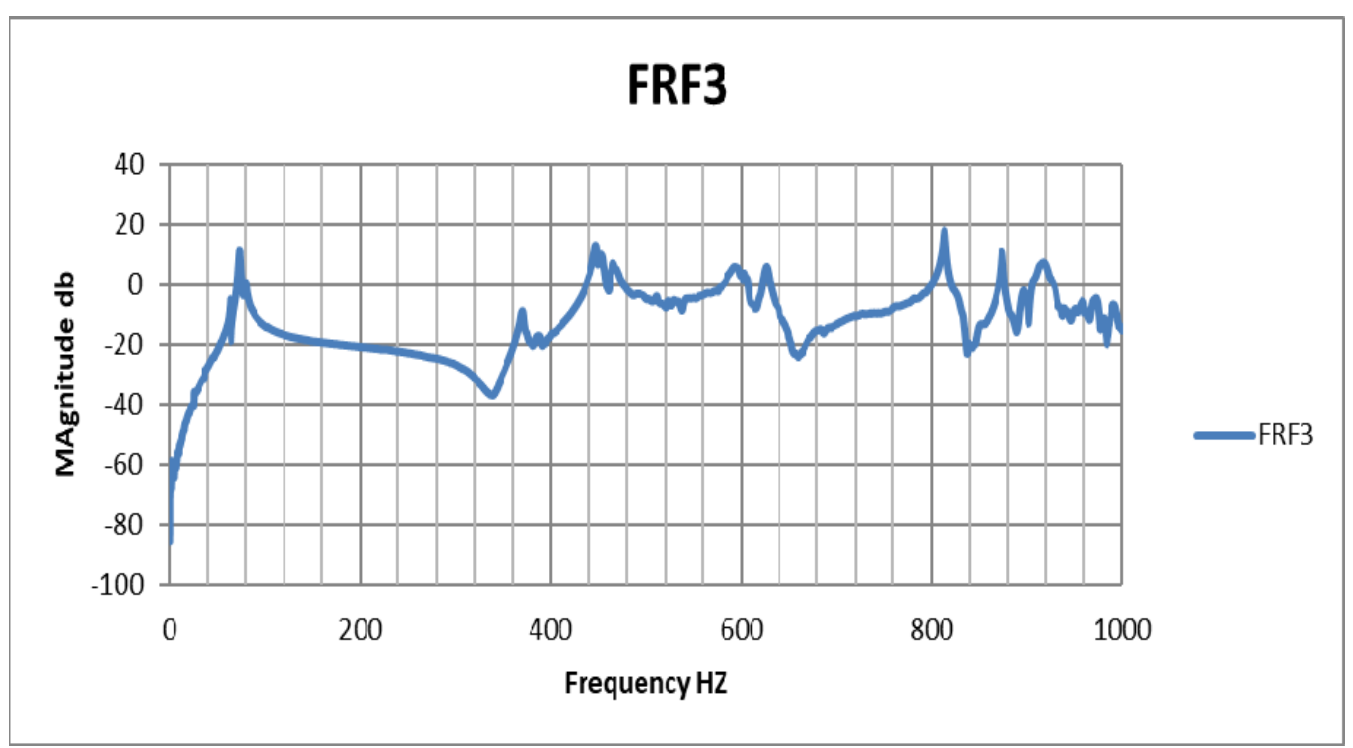

Figure 19 - Frequency response function of the damaged beam at the third point

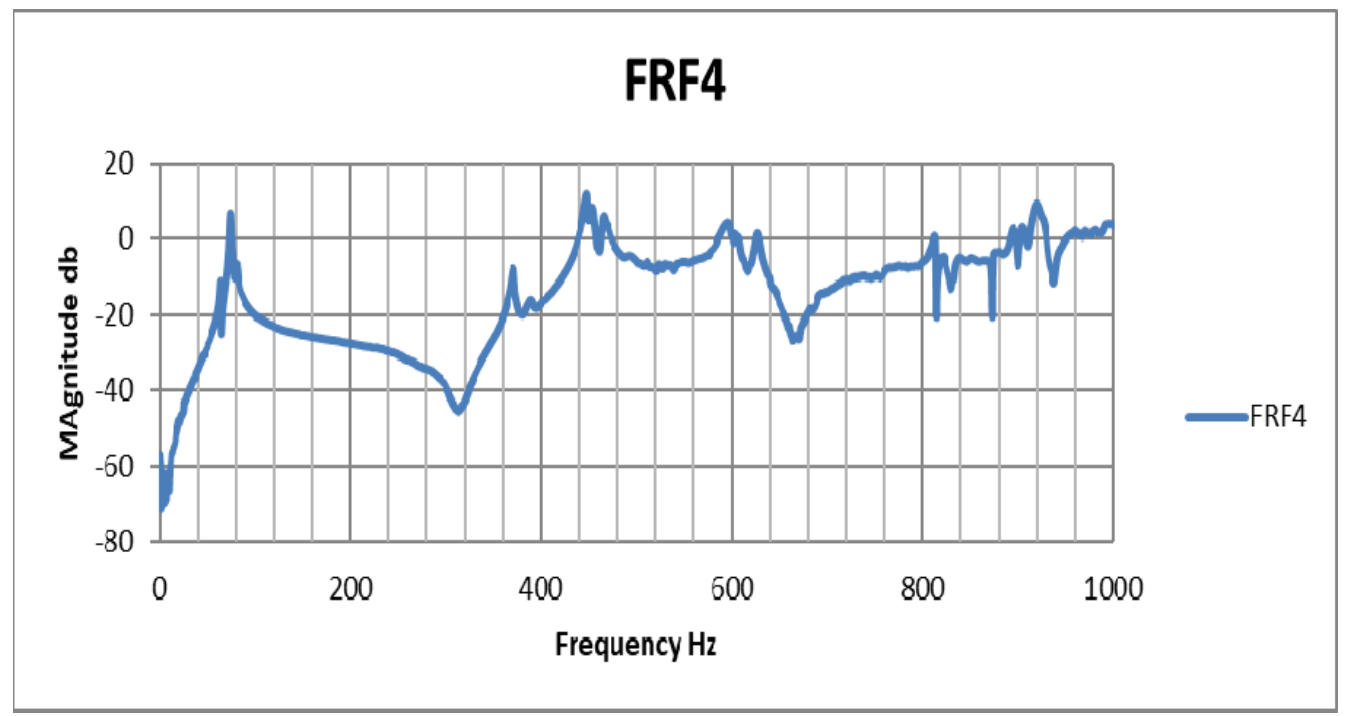

Figure 20 - Frequency response function of the damaged beam at the fourth point

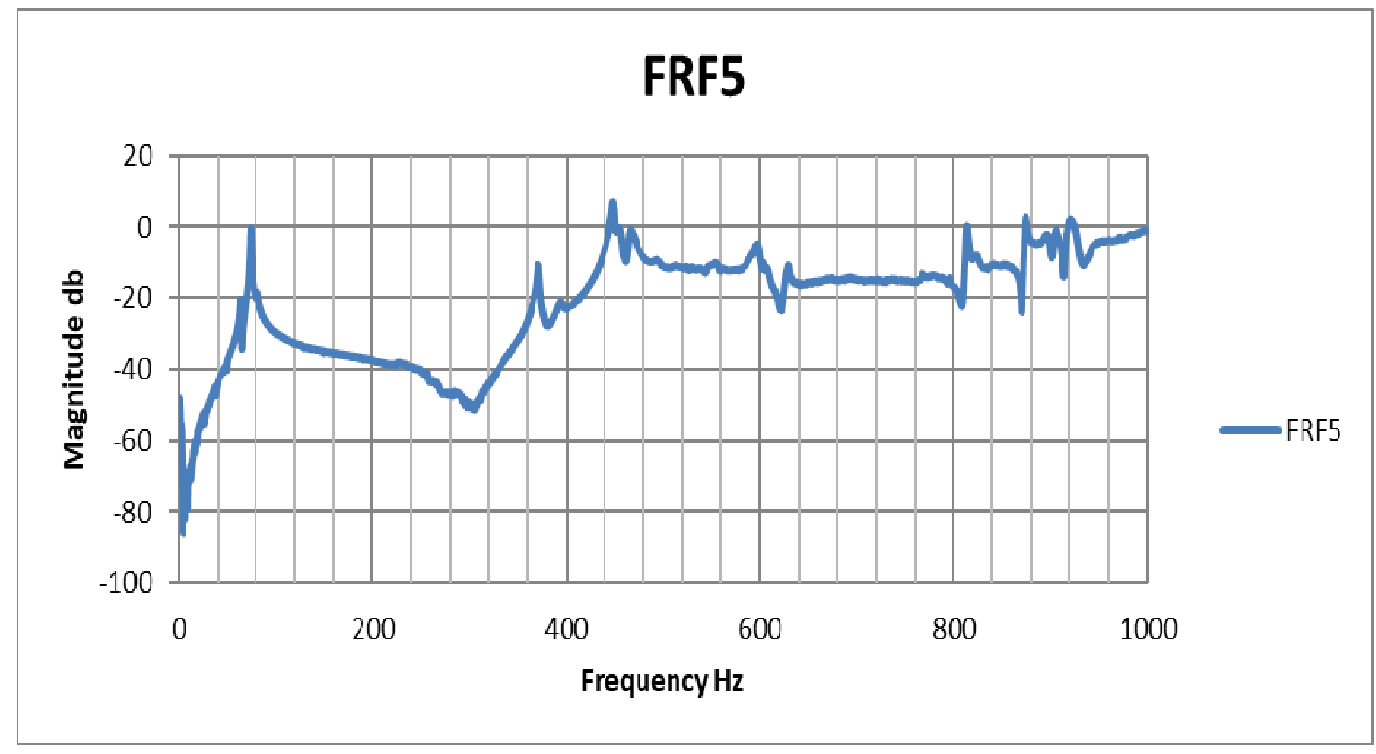

Figure 21 - Frequency response function of the damaged beam at the fifth point 
The same process explained to extract the first mode of the healthy beam is used for the damaged beam. Figure 4-8 shows the extraction of the 5th and 6th mode of the damaged beam using the NLLS1 method.

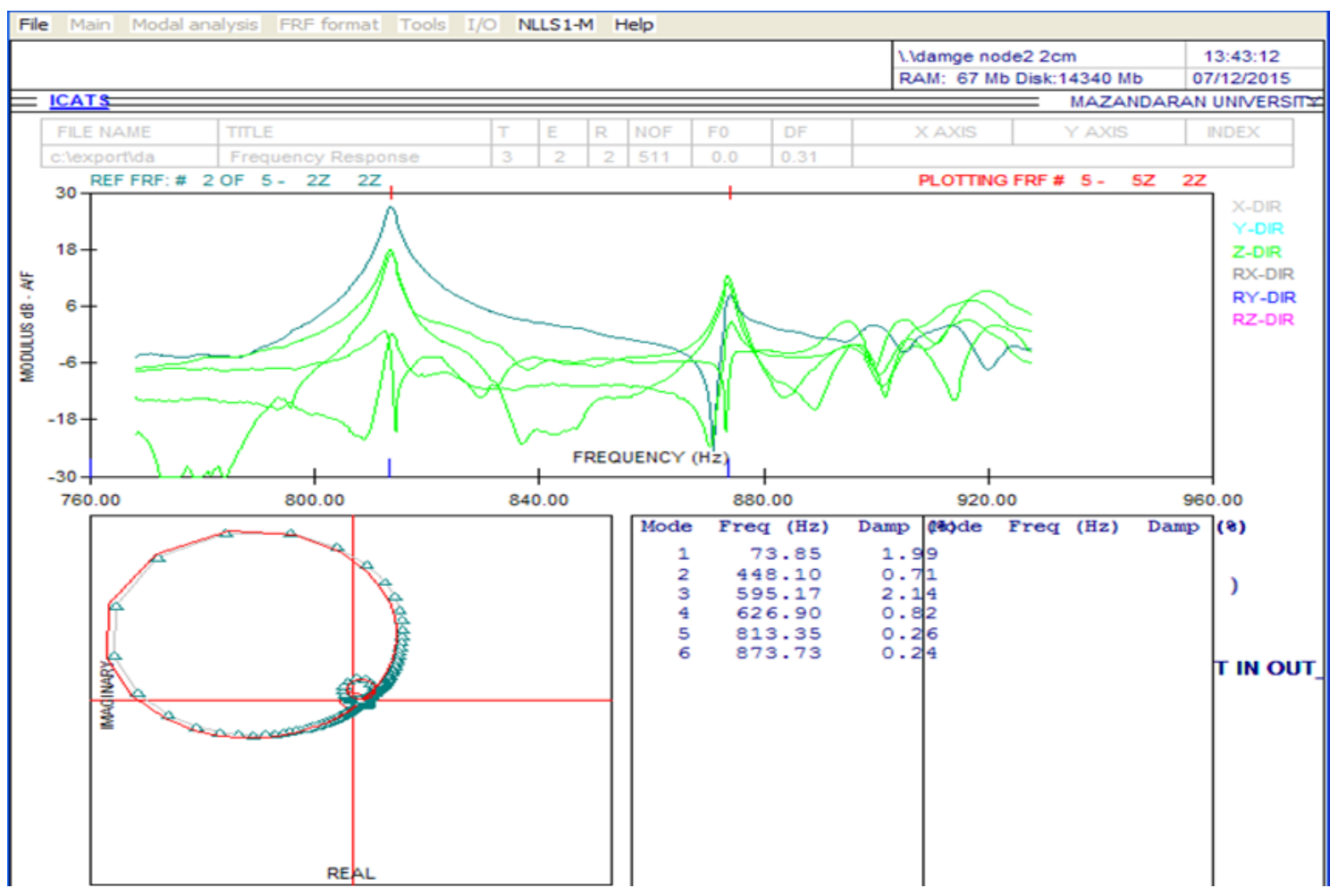

Figure 21 - Extraction of the 5th and 6th modes of the damaged beam

Table 3. Results of damaged beam modal analysis in the first damage scenario

\begin{tabular}{|l|l|l|l|l|}
\hline Mode No. & NLLS1 & NLSS2 & GRFM & Considered \\
\hline & Frequency & Frequency & Frequency & Frequency \\
\hline 1 & 73.85 & 73.65 & 73.81 & 73.77 \\
\hline 2 & 448.1 & 448.03 & 448.06 & 448.06 \\
\hline 3 & 595.17 & 595.13 & 595.35 & 595.22 \\
\hline 4 & 626.9 & 626.65 & 626.72 & 626.82 \\
\hline 5 & 813.35 & 813.35 & 813.36 & 813.35 \\
\hline 6 & 873.73 & 873.23 & 906.12 & 884.36 \\
\hline
\end{tabular}

Scenario II: Damage to the Second and Fourth Elements

In order to examine the ability of the written program to locate more than one damage with various intensities, the second damage scenario is defined. In this case, as shown in Fig.22, two parts of the beam are damaged by different intensities and a modal testing is carried out on it. 


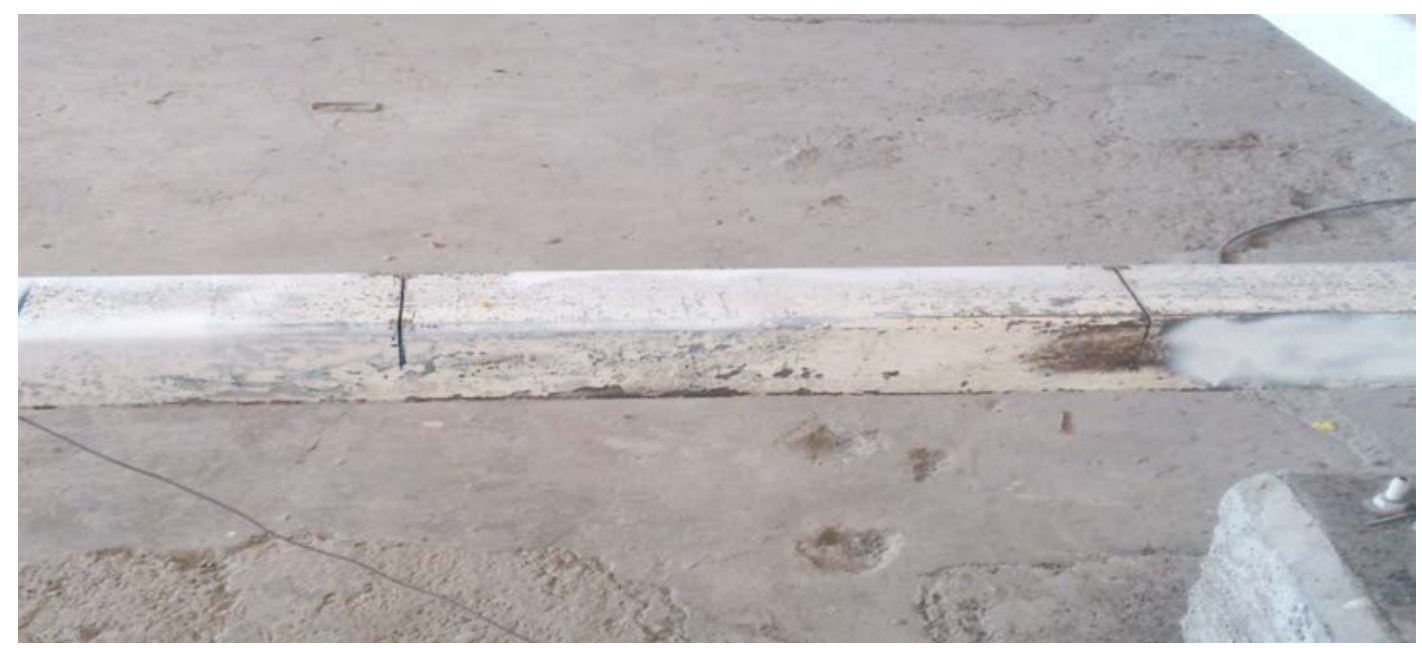

Figure 22 - Second damage scenario

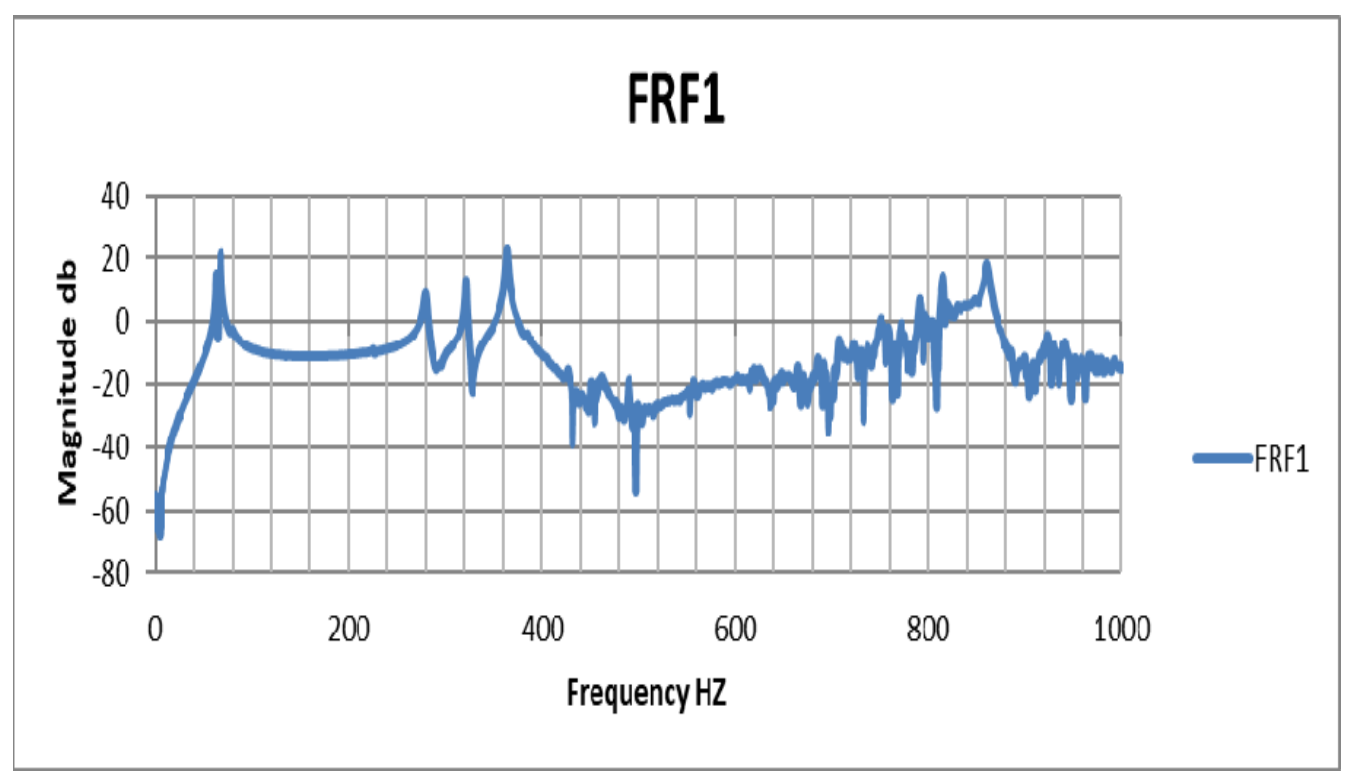

Figure 24 - Frequency response function of the damaged beam at the first point

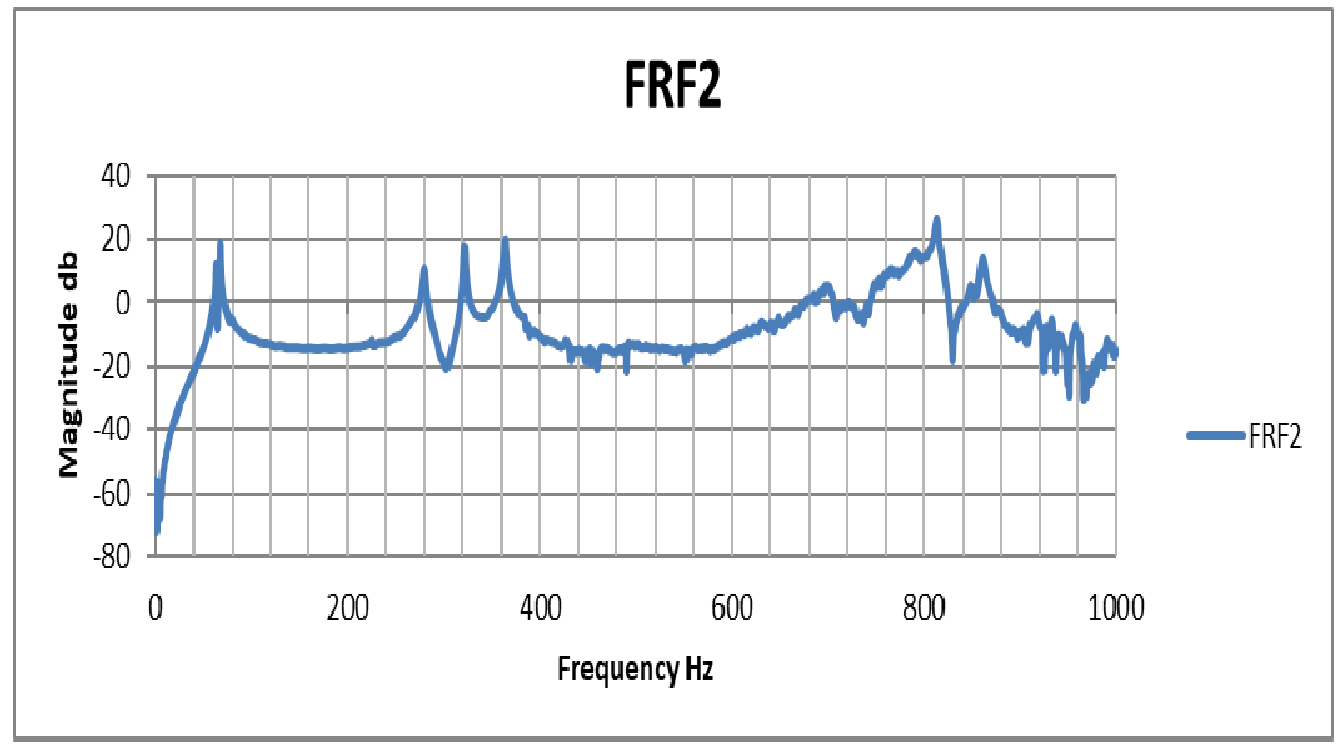

Figure 25 - Frequency response function of the damaged beam at the second point 


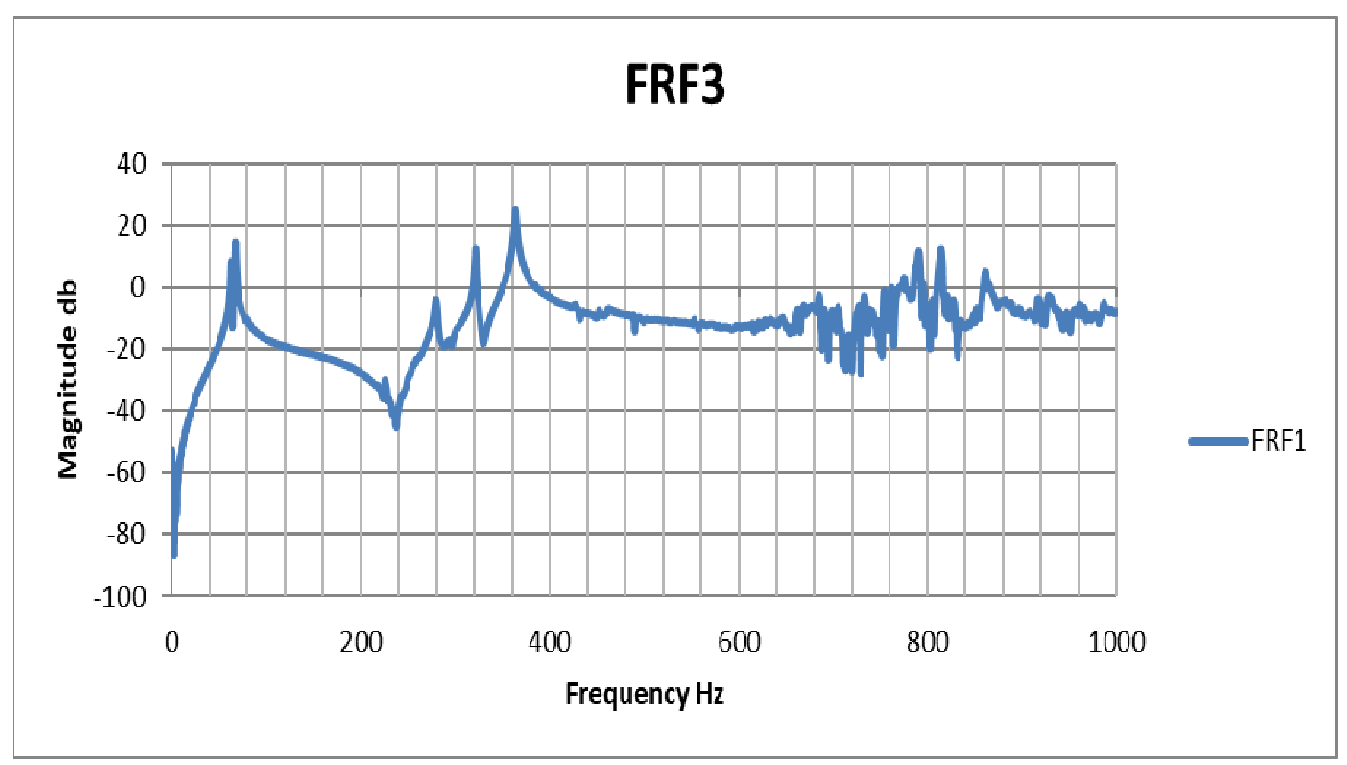

Figure 26 - Frequency response function of the damaged beam at the third point

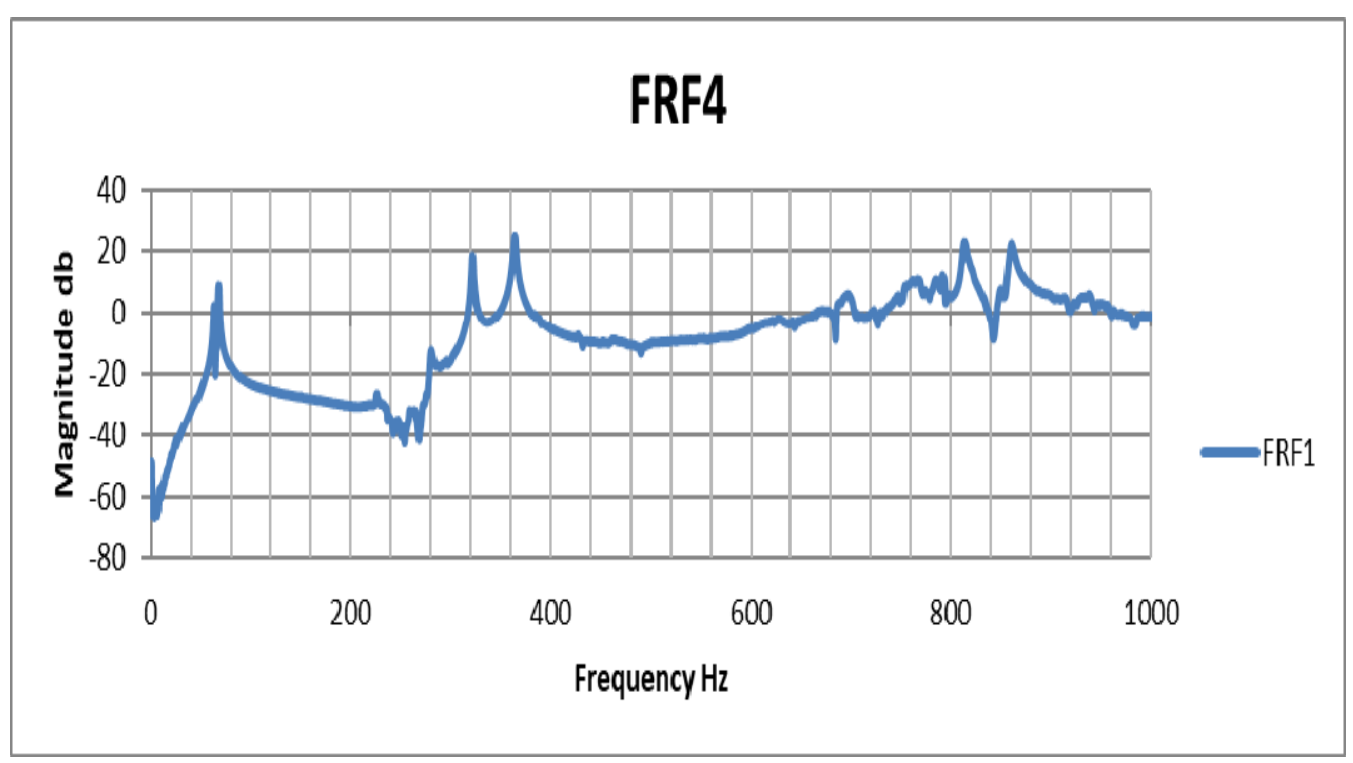

Fig. 27- Frequency response function of the damaged beam at the fourth point

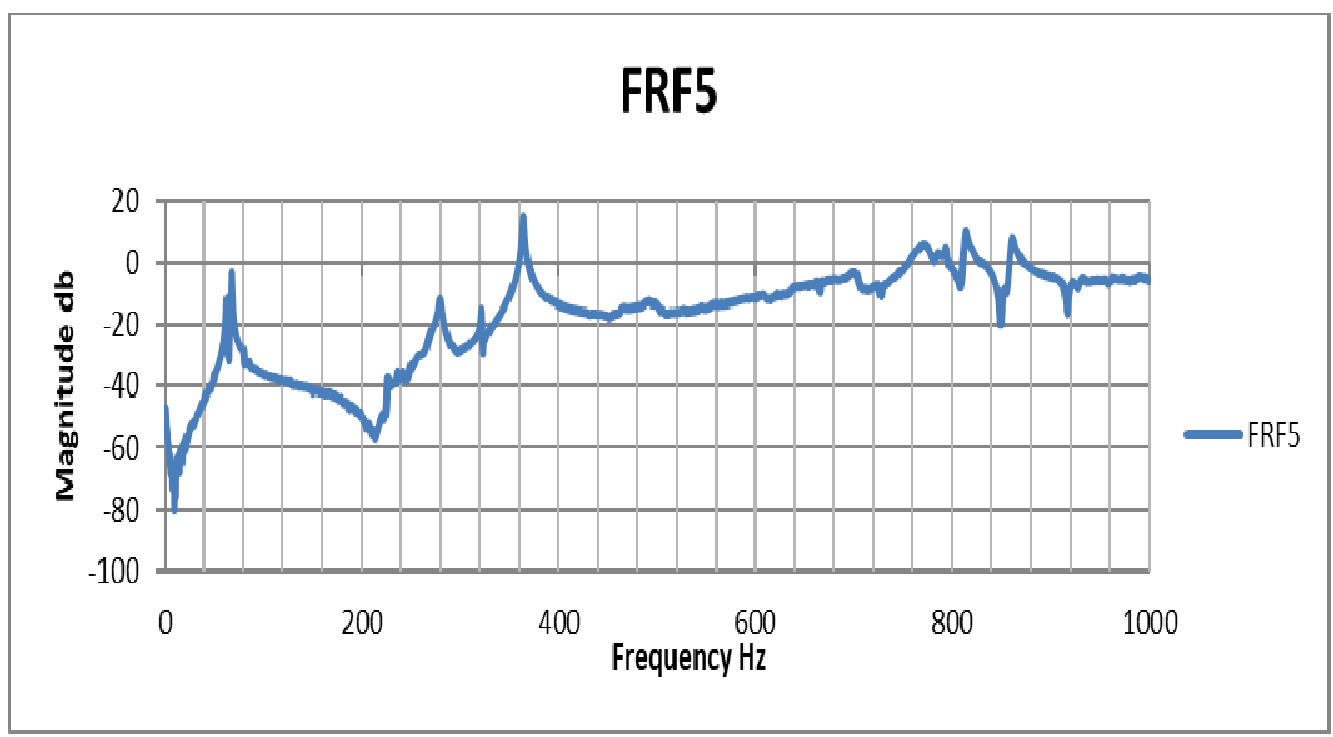

Figure 28 - Frequency response function of the damaged beam at the fifth point 
Another example is shown in Fig. 29. It shows the simultaneous extraction of the first, second, and third modes using the NLLS1 method. The three mentioned modes are in the selected frequency range of $240 \sim 400 \mathrm{~Hz}$ and their corresponding Nyquist plot includes three circles.

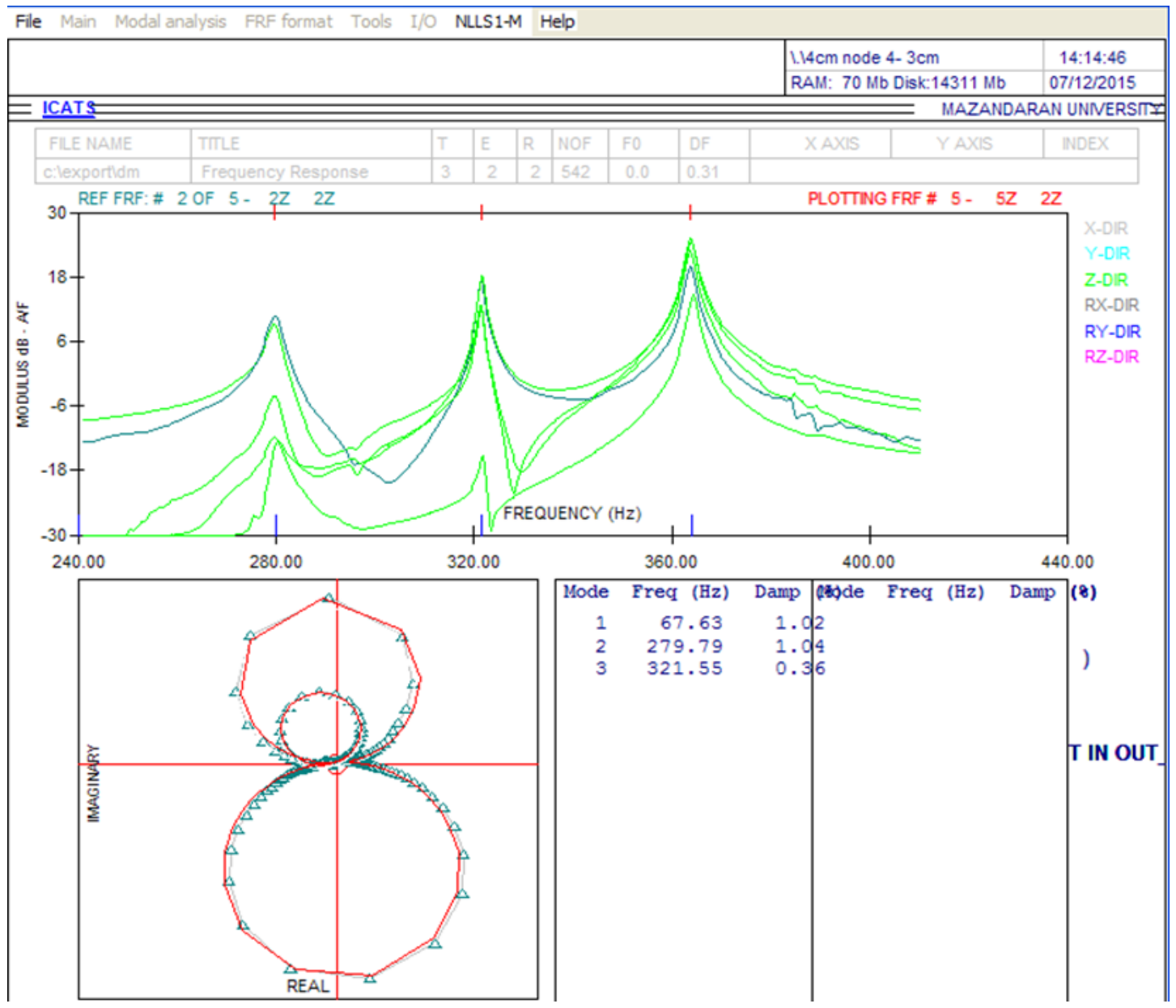

Figure 29: Simultaneous extraction of the first, second, and third modes using the NLLS1 method

The results of the empirical modal analysis of the cantilever beam using the NLLS1 method are summarized in Table 4-3. The frequencies obtained from different methods are well consistent with each other. Since different assumptions have been used in each of these methods, consistency of frequencies ensures that the empirical modal analysis phase is properly performed.

\section{3-4-2-4 Damage detection}

To identify the position and intensity of the damage, a program is written in Matlab software. The program is written in such a way that it asks the properties of the cantilever beam from the user and it detects the damaged elements at each repetition. It is observed that as the program is repeated more, it detects the position and intensity of the damage more accurately.

Table 5. Results of the damaged beam modal analysis in the second damage scenario

\begin{tabular}{|l|l|l|l|l|}
\hline Mode No. & NLLS1 & NLSS2 & GRFM & Considered \\
\hline & Frequency & Frequency & Frequency & Frequency \\
\hline 1 & 67.63 & 66.95 & 67.58 & 67.39 \\
\hline 2 & 279.79 & 279.65 & 279.52 & 279.65 \\
\hline 3 & 321.55 & 321.54 & 321.2 & 321.43 \\
\hline 4 & 395.36 & 396.01 & 398.23 & 396.53 \\
\hline 5 & 808.78 & 809.12 & 804.9 & 807.63 \\
\hline 6 & 850.21 & 851.01 & 851.1 & 850.74 \\
\hline
\end{tabular}




\section{1-3-4-2-4 Damage detection in the first scenario}

An example of the program output graphs in the second, sixth, tenth, fifteenth, twenty seventh and fifth repetitions are presented below. The fitness value begins from 25318.4602 in the first generation and reaches $1.4931 \mathrm{e}-09$ in the last generation, indicating a very good fit of analytical and empirical natural frequencies in the last generation.

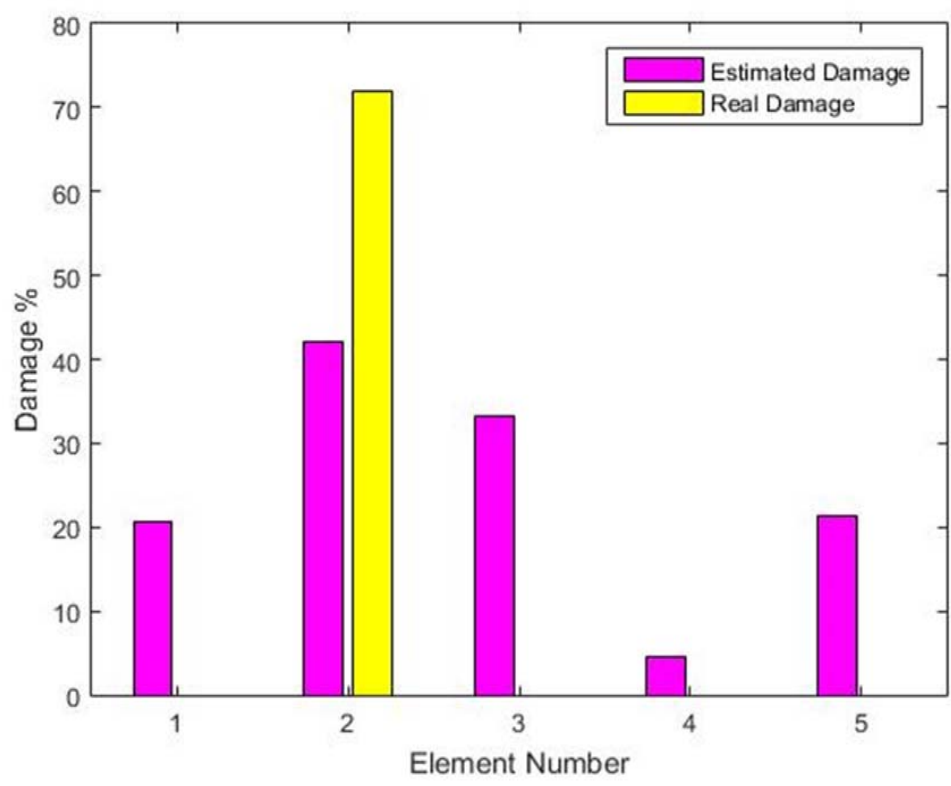

The second repetition

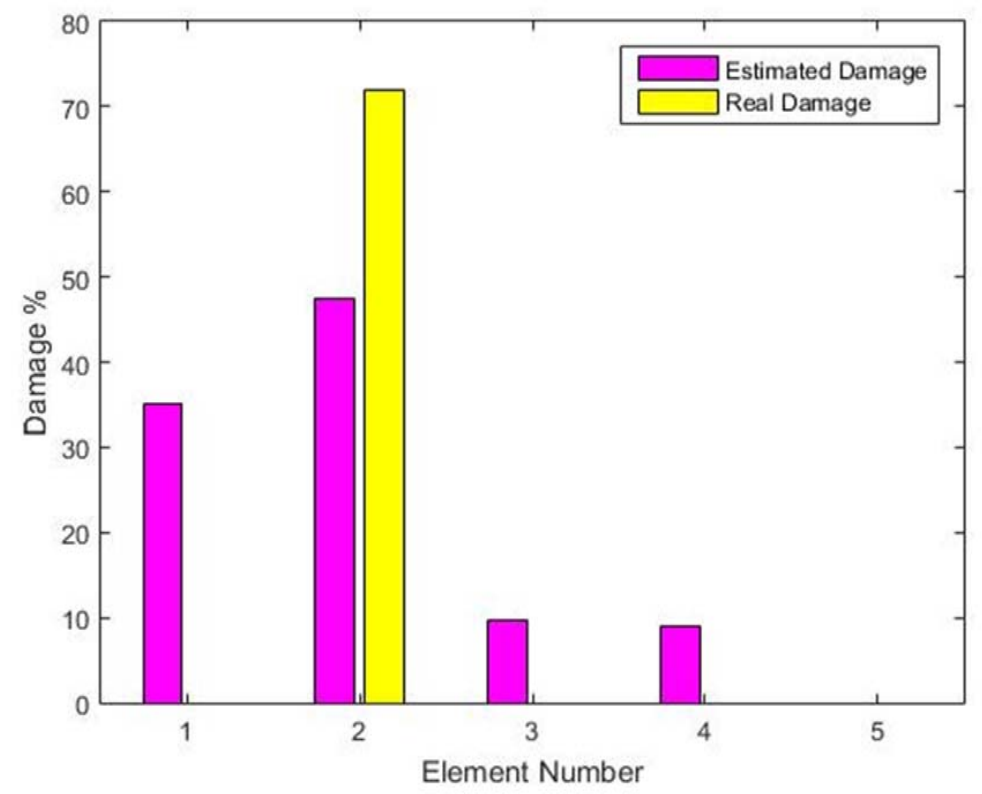

The sixth repetition 


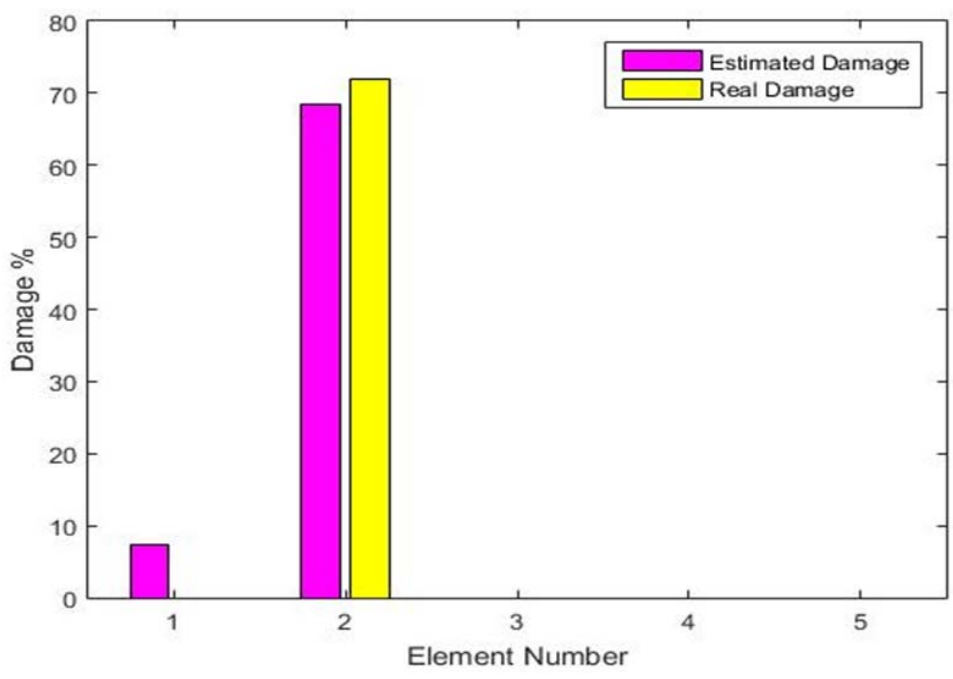

The tenth repetition

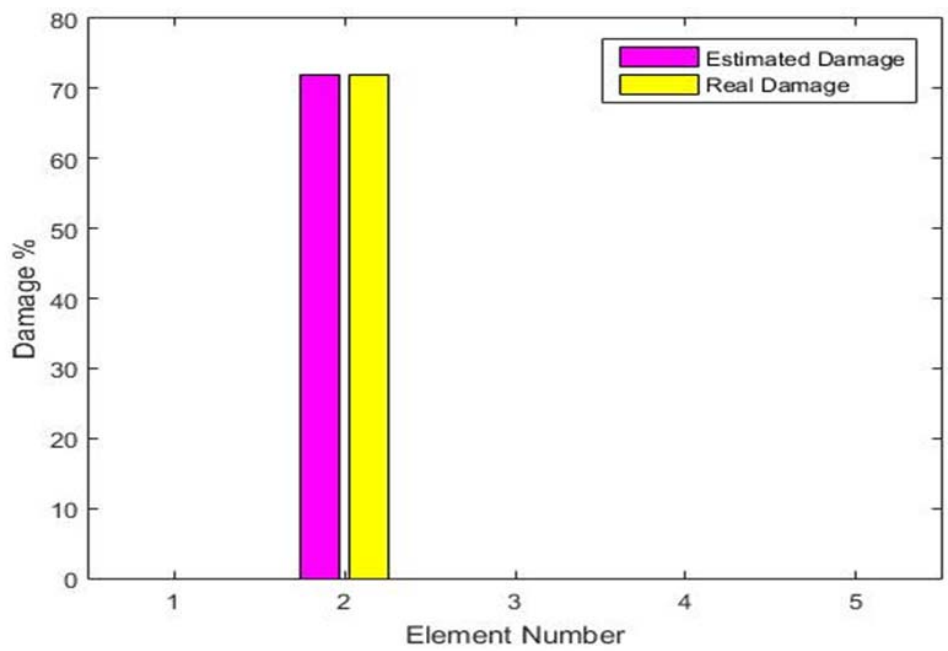

The fifth repetition

Figure 30. The elements detected in the twenty-seventh repetition

In the above graphs, the yellow bar denotes the damage to the cantilever beam in the first scenario and the purple bar actually shows the search algorithm for damage in different repetitions. It is observed that after generation for 15 times, the convergence required for the damage detection is obtained in the first scenario. This doing took 33 minutes using a computer with a 4.7GHZ CPU and 16 GB RAM. 


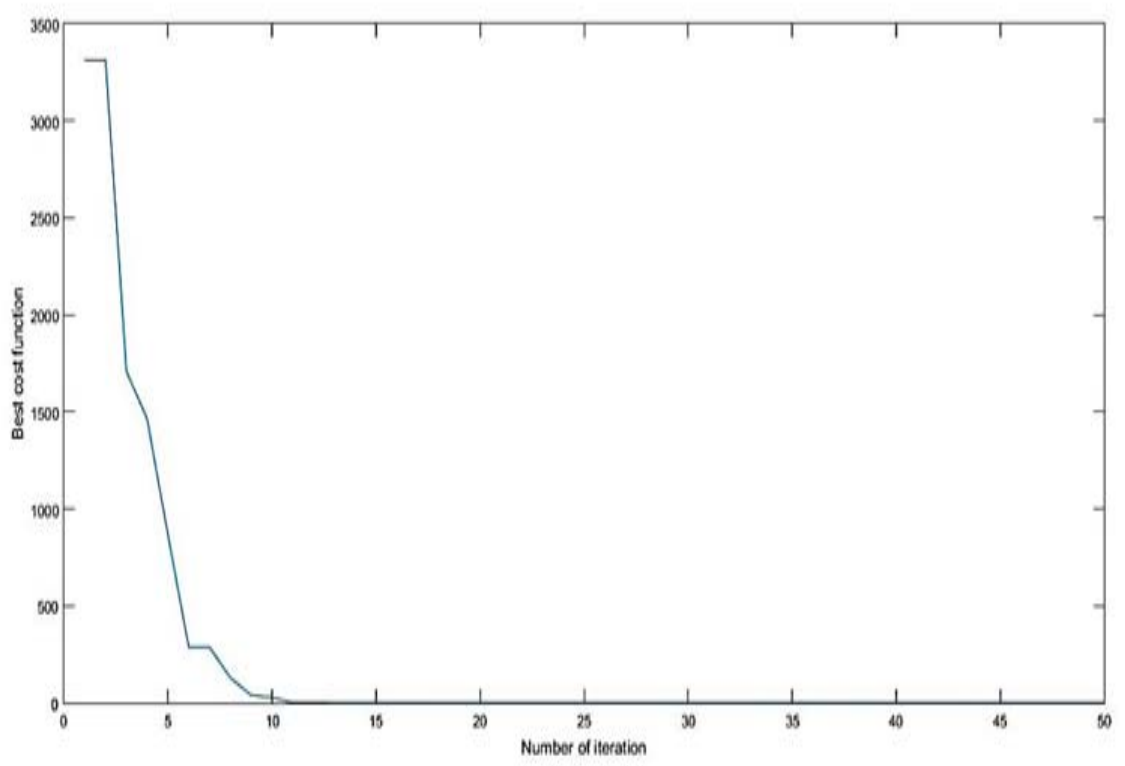

Figure 31. The values of the objective function in different repetitions

In Fig. 4-16, it can be seen that the written program detects the position and intensity of the damage in the first scenario with a very good approximation only after 15 repetitions.

\section{2-3-4-2-4 Damage detection in the second scenario}

Given that in this case, the beam is damaged at two point with different percentages, in order to expedite the process and improve the accuracy of the program, the populations of each generation are considered twice the first scenario. As in the previous example, an example of the program output graphs in the first, fifth, tenth, fiftieth and hundredth repetitions are presented below. It is observed that as the program is repeated more, it detects the position and intensity of the damage more accurately. In this scenario, in addition to detecting the position of damage in the second and fourth elements, the program also considers a very small amount of damage to the third element of the beam. Due to its negligible amount as much as $1.95 \%$, it can be ignored. Also, in order to validate the program, it is again implemented with a higher initial population and more generations. In this case, as previously anticipated, a third element is shown with very little damage, indicating the ability of the program and the strength of the Genetics algorithm.

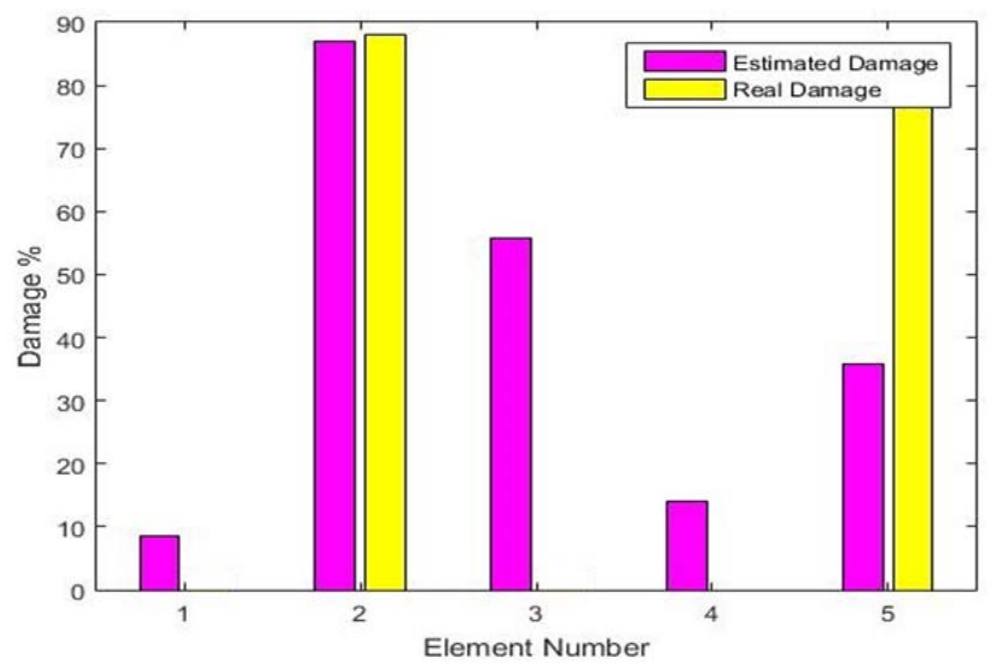

The first repetition 


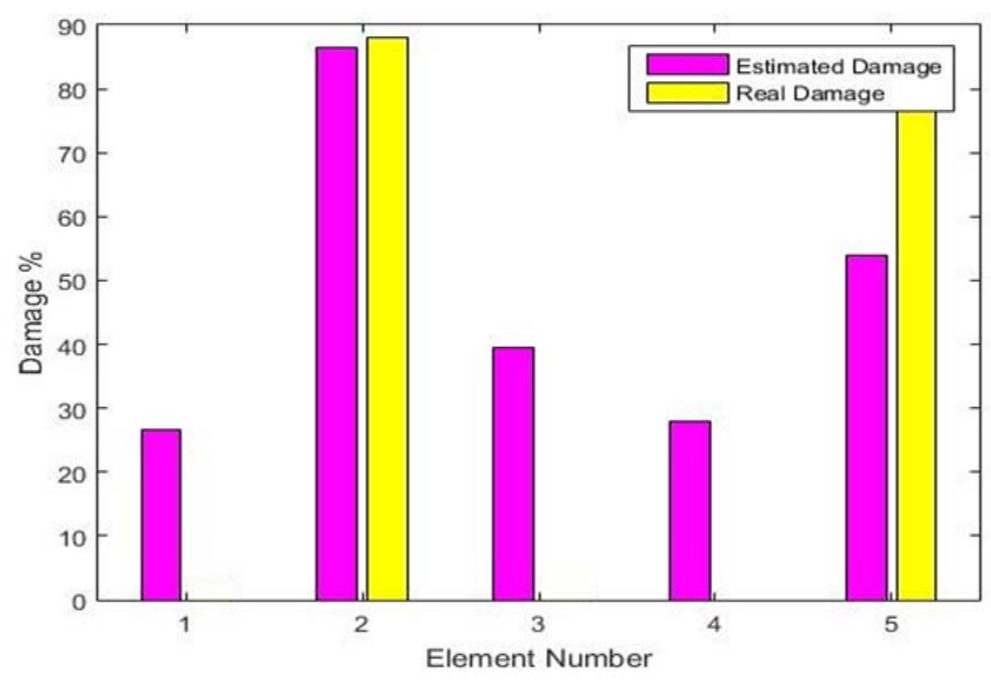

The fifth repetition

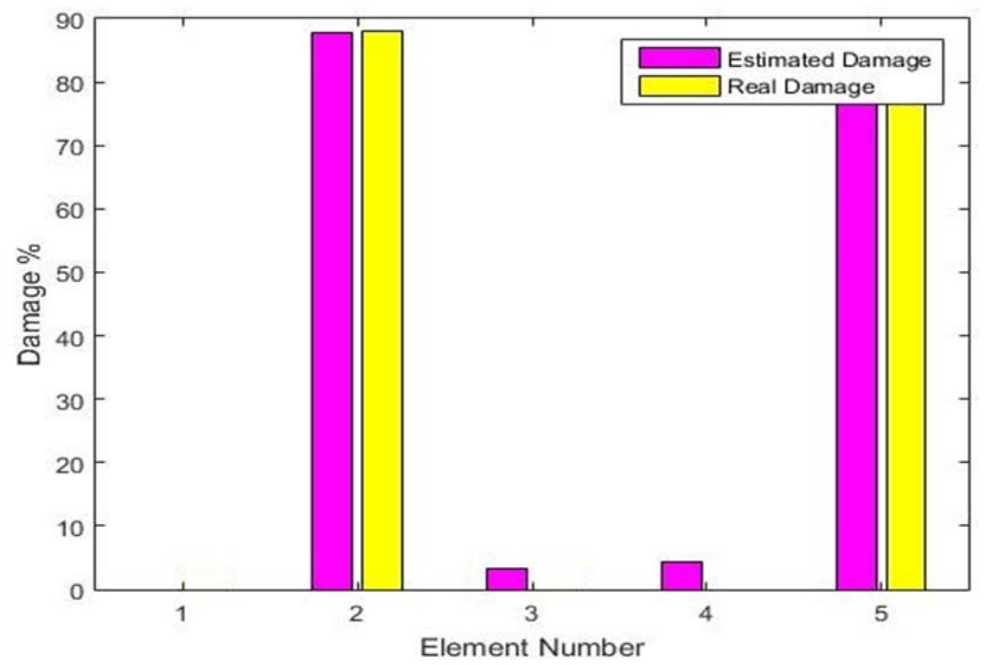

The tenth repetition

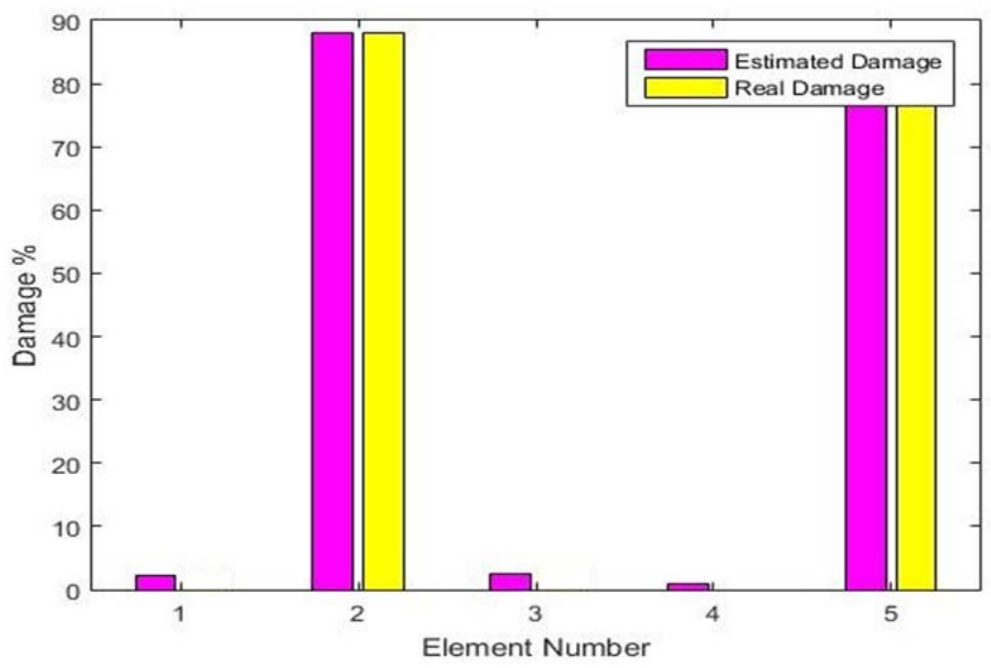

The fiftieth repetition 


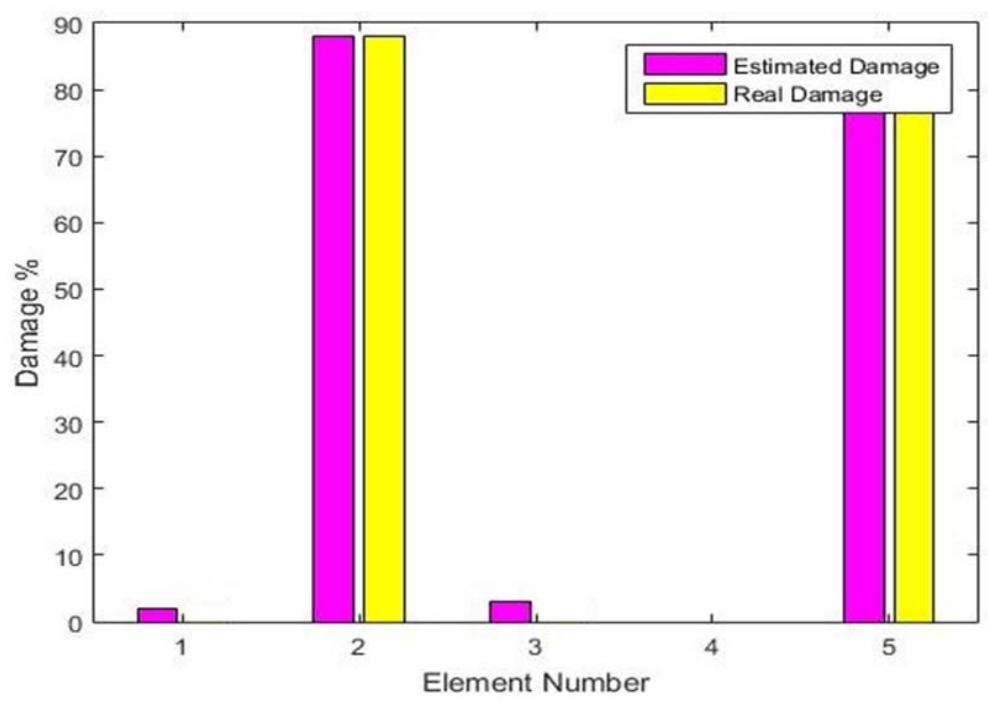

Figure 32 - The elements detected in the hundredth repetition

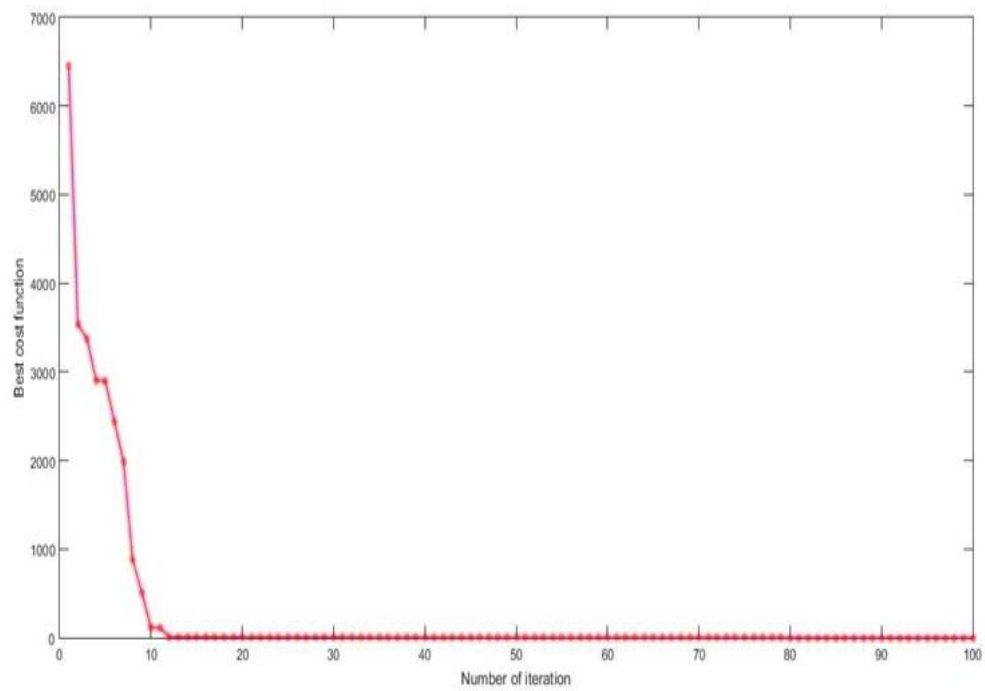

Figure 33. The values of the objective function in different repetitions

It is observed that as the program is repeated more, the damage is detected more accurately and its position in much closer to the real position. In this scenario, in addition to determining the position of damage in the second and fourth elements, the program also considers a very small amount of damage in the first and third elements of the beam. Due to its negligible amount as much as $1.95 \%$, it can be ignored. Also, in order to validate the program, it is again implemented with a higher initial population and more generations. In this case, as previously anticipated, a third element is shown with very little damage, indicating the ability of the program and the strength of the Genetics algorithm.

\section{2-5-Conclusion}

In present study, it has been shown that using the frequency response function data and model updating, one can find the position and intensity of damage in a cantilever beam. The only thing required for the purpose of detecting a damaged beam is a column, or a row of the frequency response function matrix. According to the outputs of the program, it can be concluded:

- If the cantilever beam is damaged only at a point, present study indicates that the position and intensity of the damage can be determined with great accuracy using the frequency response function and updating the model.

- For a cantilever beam with five elements and two damaged points, the program, in addition to damaged points, provides a small of damage to the neighboring elements of the damaged points. 
- To completely assure the program output, it is better that the number of reference responses is more than the number of unknowns, which are the number of damaged elements. For the cantilever beam studied. If the number of reference responses for model updating is more than twice the number of unknowns, the position and intensity of the damage are determined well.

- The position of the hammer blow considering the natural frequencies (which is an inherent characteristic of the frequency response function) does not generally have a negative effect on the result. Therefore, it is possible to use any point that makes the implementation of the modal experiment easier, but it is recommended to consider the largest distance between the positions of the blow and accelerometer and the support.

\section{References}

[1] Los Alamos National Laboratory Report,A Review of Structural HealthMonitoring Literature.. LA-13976-MS. 2003

[2] Chan Ghee Koh and Michael john Perry, structural identification and damage detection using Genetic algorithms, department of civil engineering national university of Singapore ,2010.

[3] Doebling, S.W., et al., Damage identification and health monitoring of structural and mechanical systems from changes in their vibration characteristics: a literature review, Los Alamos National Laboratory, 1996

[4] Chang, P., Flatau, A., Liu, C. , Review Paper: Health Monitoring of Civil Infrastructure. Structural Health Monitoring, 2(3): p. 257267, 2003.

[5] "Modal Analysis” Book by Jimin He and Zhi-Fang Fu ,1995

[6] Hjelmstad, K. D., Wood, S. L., and Clark, S. J. "Mutual residual energy method for parameter estimation in structures." J. Struct. Engrg., 118(1), 223- 242,1992.

[7] I.Y. Choi, J.S. Lee, E. Choi, H.N. Cho, "Development of elastic damage load theorem for damage detection in a statically determinate beam”, Computers \& Structures,vol. 82, pp. 2483-2492, 2004.

[8] F. Bakhtiari-Nejad, A. Rahai, A. Esfandiari, “A structural damage detection method using static noisy data”, Engineering Structures, vol. 27, pp. 1784-1793, 2005.

[9] Davoudi, Mohammad Reza and Hamed Teymouri, 2014, Determination of the position and intensity of damage in space structures using frequency response function, Fourth National Conference on Space Structures, Tehran, Tehran University.

[10] Mostafavan, A., Dissertation, Babol Noshirvani University of Technology, "Finite Element Model Upgrading of the Double-Layer latticed Structure with Nodular System Using Empirical Dynamic Responses", 2012. 